\title{
Perspectives on Molecular-Level Understanding of Thermophysics of Liquids and Future Research Directions
}

\author{
Department of Mechanical Engineering, \\ Massachusetts Institute of Technology, \\ Cambridge, MA 02139 \\ e-mail: gchen2@mit.edu
}

Gang Chen
This article provides the author's perspectives on the current molecular-level understanding of thermophysical properties and transport processes in liquids. After illustrating peculiarities of the thermophysical properties of some common liquids using experimental data on their specific heat, thermal conductivity, and viscosity, the article moves on with a summary of existing molecular pictures and theoretical approaches on liquids, followed with elaborations on different models developed for the specific heat, thermal conductivity, and viscosity. The review shows that current understanding of thermophysical properties of liquids is still poor and theoretical tools to study them are not well developed. The article provides personal views of the author on what is missing in current theories. Furthermore, it explains underlying mechanisms for some experimental observations and suggests potential directions of future research.

[DOI: $10.1115 / 1.4052657]$

\section{Introduction}

Despite thermal systems extensively using liquids, fundamental understanding of the thermodynamic and transport properties of liquids is far from complete. In contrast, molecular-level understanding of thermophysical properties of gases is well-established, building on the statistical thermodynamics and the Boltzmann transport equation [1]. Thermophysical properties of crystalline solids can be similarly understood based on the phonon gas picture [2]. Over the last few decades, extensive studies on micro and nanoscale heat conduction in solids have led to great insights on transport pictures and advances in extreme thermal conductivity materials [3-7]. Given the prevalent use of liquids in thermal systems, it is highly desirable that we have better molecular-level understanding of their thermodynamic and transport properties.

In gases, the collision time between molecules is short compared to the free traveling time between molecular collision events. This fact allows the kinetic theory to focus on the kinetic and internal energy of individual molecules, treating the collision as perturbations to the molecules' trajectories, which can be described using the Boltzmann transport equation. In solids, although atomic interactions via potential energy are important, the atoms oscillate around their equilibrium positions with small amplitudes such that harmonic-oscillator model can serve as a good starting point. The quantization of collective oscillation modes led to the phonon picture. For crystals, due to the regular arrangements of the atoms, phonon modes extend across the entire crystal. Their interactions via higher-order anharmonic force can be treated as perturbations that change phonons' trajectories, whose transport can again be described by the Boltzmann transport equation as Peierls had shown [8], under proper conditions [5].

There are two major difficulties in the physical understanding and the mathematical treatment of liquids' thermodynamic and transport properties. (1) Unlike solids, the atomic positions in liquids are not fixed, and (2) due to the close proximity of the atoms, their interactions via potential energy are strong. The ideal gas law is not valid for thermodynamic properties of liquids; nor is the Boltzmann transport equation for their transport properties. Many luminaries had worked on better understanding and

Contributed by the Heat Transfer Division of ASME for publication in the Journal of Heat Transfer. Manuscript received September 3, 2021; final manuscript received September 24, 2021; published online November 9, 2021. Assoc. Editor: Portonovo S. Ayyaswamy. treatment of liquids in the past. One line of thought to understand transport in liquids is by modifying the Boltzmann theory on gas, as represented by the effort of Enskog [9]. As we will discuss later, this line of research ran into considerable obstacles. Another line of research was pioneered by Einstein in his effort to determine the molecular size by studying the Brownian motion [10]. This approach evolved in two directions: (1) reduction of the Liouville equation describing the $N$-particle distribution function in the system to lower-order particle distribution functions and (2) the linear-response theory based on the perturbation solution of the Liouville equation for the time-evolution of the $N$-particle distribution function [2]. The former forms the basis of prevailing hydrodynamic treatment of liquids [11], and the latter is the foundation of molecular dynamics simulations, a major tool used nowadays in studying liquids. The third line is to start from the solid picture [12], which arguably can be traced back to Maxwell in his study leading to the famous Maxwell velocity distribution of gas molecules [13]. This line of research did not gain enough traction but has been seeing resurgence over the last decade [14].

I have not done much work in liquids, but have always been curious about them, starting from the time I was writing a book on nanoscale heat transfer [2] until more recently when I was planning to shift my research focus to soft materials. Professor Ayyaswamy has been very persistent in asking me to contribute an article to the Journal of Heat Transfer. Ironically, I get more time during a stressful period in my life. So, I accepted Professor Ayyaswamy's invitation and decided to challenge myself by writing an article reviewing the current understanding of thermodynamic and transport properties of liquids. This article summarizes what I have learnt so far. The properties to be discussed include specific heat, thermal conductivity, and viscosity, with some preference on thermal conductivity. Due to the vast number of studies done over 100 years in trying to understand liquids $[11,15]$, it is impossible for me, nor have I attempted, to review all important literatures. I took an approach of not avoiding controversies and not hiding my personal opinions, despite the fact that they are still nascent as I am a beginner. In being so open, my goal is to stimulate others to tackle challenges confronting this topical area since currently the ranks of us working on fundamental thermophysical properties of liquids in the heat transfer community are small.

The rest of this article is arranged as follows. Section 2 presents experimental data on specific heat, thermal conductivity, and viscosity of selected liquids to illustrate some puzzles in understanding liquids. Section 3 explains key pictures and models 


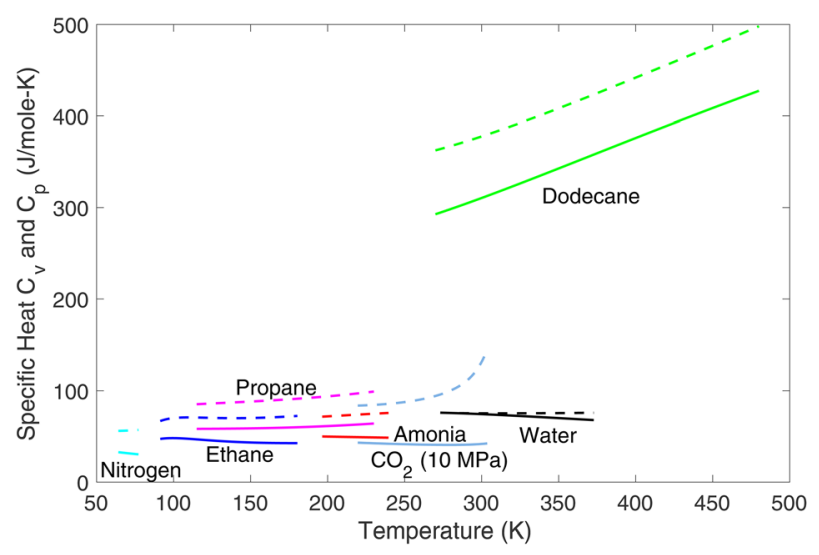

(a)

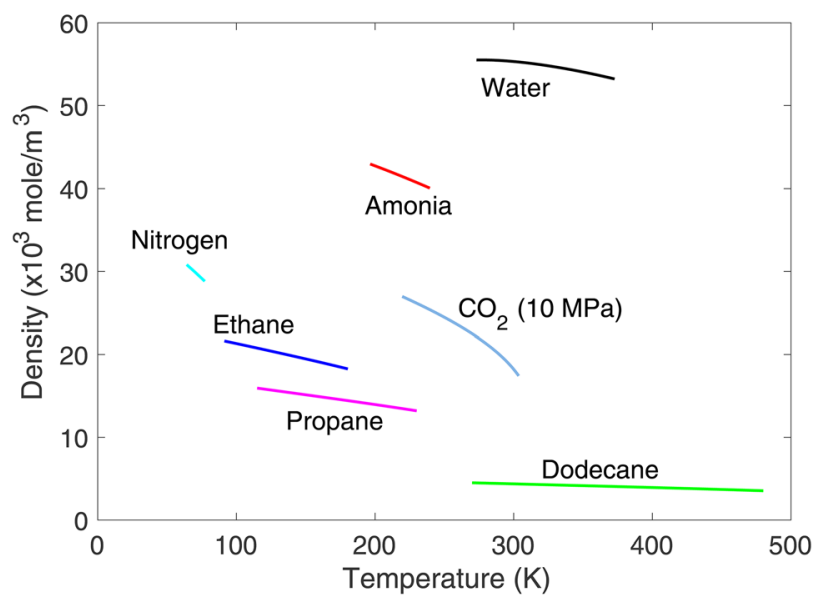

(b)

Fig. 1 Temperature dependence of (a) constant volume $\left(C_{v}\right)$ (solid lines) and pressure $\left(C_{p}\right)$ (dashed lines of same temperature range for each species) specific heat, and $(b)$ density. All properties are at atmospheric pressure except $\mathrm{CO}_{2}$, which is at $10 \mathrm{MPa}$.

researchers have developed on liquids. Sections 4-7 review models developed to explain the trends, especially the temperature dependence of specific heat, thermal conductivity, and viscosity for liquids, coupled with elaboration on gaps in our understanding. In some places, I offer my own views on missing links and potential mechanisms. Finally, the article concludes with some speculations on potential future research directions.

\section{Peculiarities of Liquid Thermodynamic and Transport Properties}

The liquid phase of a substance usually sandwiches between its solid and vapor phases over a narrow temperature range. Extensive experimental data on specific heat, thermal conductivity, and viscosity of liquids exist. Here, we show data on a few selected liquids taken from the U.S. National Institute of Standards and Technology database [16], with a special focus on peculiarities associated with their thermophysical properties.

Specific Heat and Density. Unlike solids whose specific heats increase with temperature and then saturate, the constant volume specific heat of most inorganic liquids decreases, while that of the organic liquids increases with increasing temperature, as shown in Fig. 1(a). The specific heat unit chosen is based on per mole of molecules in the liquid, as we will see that molecules are more important in liquids than individual atoms. Also shown in the same figure is the constant pressure specific heat of the same liquids. In heat transfer analysis, we often assume that the constant volume and the constant pressure specific heat equal each other. Figure 1 $(a)$ shows that this assumption can be erroneous, especially near the boiling point. Density of most liquids (Fig. 1(b)) decreases with increasing temperature, except water, which has a density peak at $4{ }^{\circ} \mathrm{C}$.

Thermal Conductivity and Diffusivity. Compared to solids, whose thermal conductivities span 4 orders of magnitude at room temperature, liquid thermal conductivity falls into a narrow range, with the exception of liquid metals. From the extensive list of published data $[17,18]$, the thermal conductivity of organics at $25^{\circ} \mathrm{C}$ ranges from $0.049 \mathrm{~W} / \mathrm{m} \mathrm{K}$ of octafluopropane $\left(\mathrm{C}_{3} \mathrm{~F}_{8}\right)$ to $0.36 \mathrm{~W} / \mathrm{m} \mathrm{K}$ of formamide $\left(\mathrm{CH}_{3} \mathrm{NO}\right)$, while that of inorganics ranges from $0.044 \mathrm{~W} / \mathrm{m} \mathrm{K}$ of hydrogen iodine (HI) to $0.89 \mathrm{~W} / \mathrm{m} \mathrm{K}$ of hydrazine $\left(\mathrm{N}_{2} \mathrm{H}_{4}\right)$. Thermal conductivity of most liquids decreases with increasing temperature, but some liquids, including water, have opposite trends (Fig. 2(a)). These behaviors differ greatly from gases whose thermal conductivities usually increase with increasingly temperature. They are also different from predictable trends in solids [2]: amorphous solids' thermal conductivities usually increase while that of crystalline solids usually decrease with increasing temperature (except at lower temperature range when size effects start to limit the phonon mean free path in crystals). Also shown in Fig. 2(b) is the thermal diffusivity, $a=k /$ $\left(\rho c_{v}\right)$.

As we will discuss later, no existing theories can explain thermal conductivity of liquids completely satisfactorily. Purely empirical correlations have also been provided by different

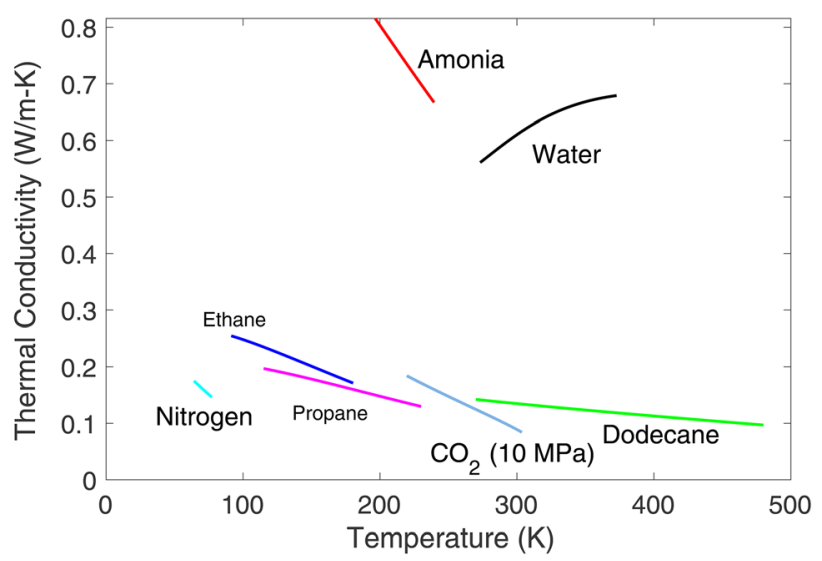

(a)

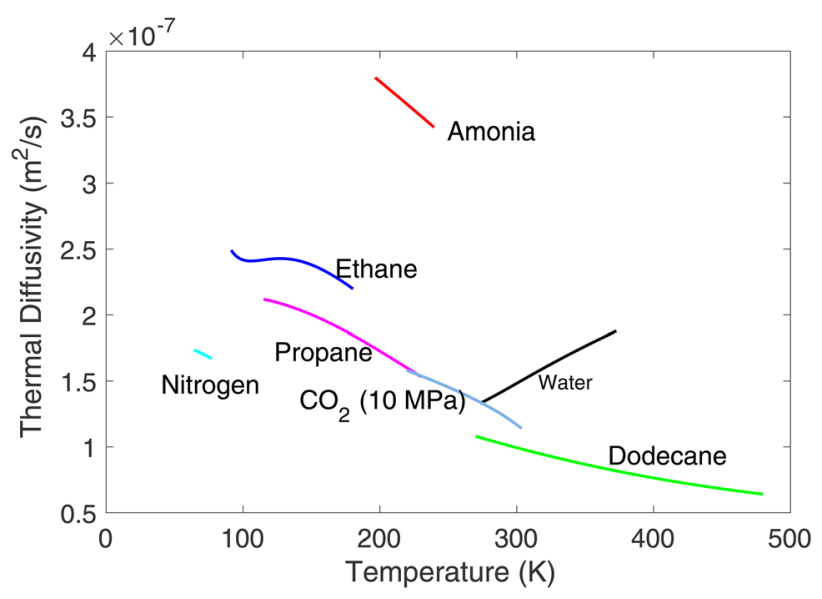

(b)

Fig. 2 Temperature dependence of (a) thermal conductivity and $(b)$ thermal diffusivity of selected liquids. All properties are at atmospheric pressure except $\mathrm{CO}_{2}$, which is at $10 \mathrm{MPa}$. 


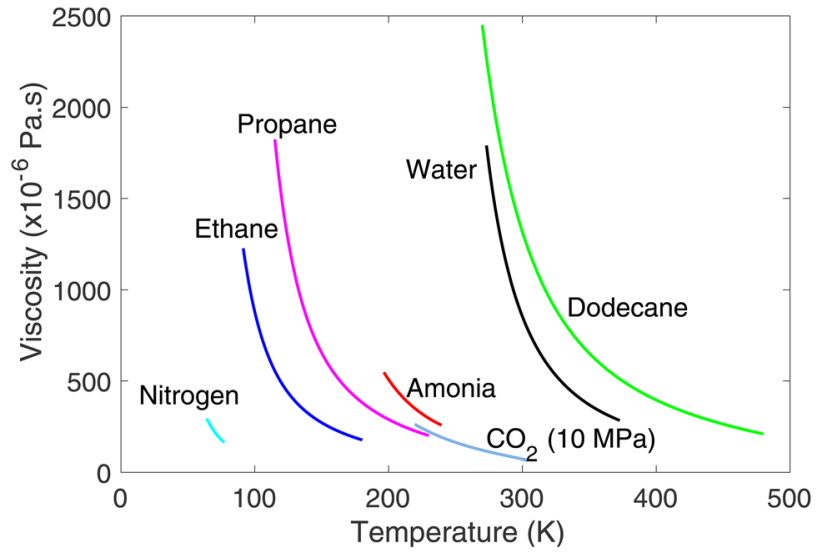

Fig. 3 Viscosity of selected liquids as a function of temperature. All properties are at atmospheric pressure except $\mathrm{CO}_{2}$, which is at $10 \mathrm{MPa}$.

researchers [18,19]. For example, the following correlation for pure liquids' thermal conductivity is often used [20]:

$$
k=\frac{A\left(1-T / T_{c}\right)^{0.38}}{\left(T / T_{c}\right)^{1 / 6}}
$$

where $T_{c}$ is the critical temperature, and $A$ is a material-dependent constant. Baroncini et al. provided correlation for binary organic liquid mixtures [21]. Yaws [18] also gave extensive data and correlations of thermal conductivity of both organic and inorganic liquids, using linear and quadratic fits.

Viscosity. Compared to the temperature dependence of the thermal conductivity, the viscosity of liquids changes much more rapidly with temperature, generally decreasing with increasing temperature (Fig. 3).

Prandtl Number. Some transport properties are related to each other [2]. For liquid, viscosity $\mu$ can often be related to molecular diffusivity $D$ by the Einstein formula, $D=k_{B} T /(3 \pi d \mu)$, where $k_{B}$ is the Boltzmann constant, and $d$ is the molecular diameter. For metals, the thermal conductivity can be related to that of electrical conductivity by the Wiedemann-Franz law $k /(\sigma T)=L$, where $k$ is the thermal conductivity, $\sigma$ is the electrical conductivity, and $L$ is the Lorentz number. For gases, the viscosity and thermal conductivity are related $k=5 k_{B} \mu /(2 m)$ for a monatomic gas, where $m$ is the gas molecule's mass. This relationship leads to Prandtl number, $\operatorname{Pr}=\nu / a=3 / 5$ for a monatomic gas which has a specific heat of $3 k_{B} / 2$ per molecule. Prandtl number values for liquids, however, change widely and depend on temperature, as shown in Fig. 4. So far, liquid thermal conductivity cannot be related to other transport properties (other than thermal diffusivity by definition).

Pressure Dependence. It is also interesting to examine the pressure dependence of the above properties. In Figs. 5(a)-5(c), we showed the pressure dependence of specific heat, thermal conductivity, and viscosity for three liquids. The specific heat can increase, decrease, or shows a minimal as a function of pressure. The thermal conductivity all increases with pressure. Viscosity of water shows a minimum, but that of ammonia and propane increases with pressure.

\section{General Pictures and Theories on Liquids}

We know that liquids are distinct from solids in that they cannot withstand shear stress. However, this statement tends to emphasize the difference between liquids and solids. In fact, glasses

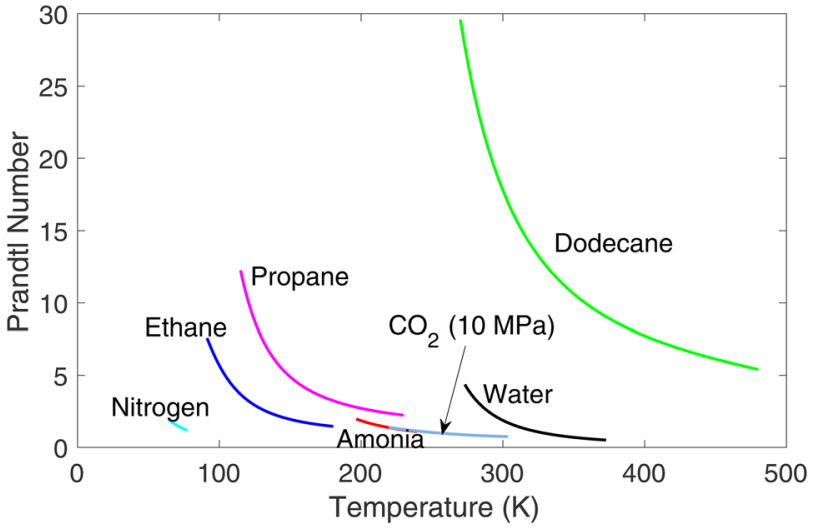

Fig. 4 Prandtl number of selected liquids as a function of temperature. All properties are at atmospheric pressure except $\mathrm{CO}_{2}$, which is at $10 \mathrm{MPa}$.

obtained from cooling of melt (rather than vapor condensation which is referred to as amorphous) are structurally and thermodynamically the same phase as their liquids [22], suggesting the differences between liquids and solids are small. Many studies and books have been devoted to understanding liquids, and significant progress has been made (see, for example, Refs. [11,12,15,23], and [24]). Despite the progress, our current ability to predict the thermodynamic and transport properties of liquids is still lacking. Below is a brief summary of different approaches to understanding liquids, loosely grouped into (1) modeling the partition function, (2) rigorous statistical formulation, (3) simulation, and (4) phonon theory. Commentary on water and electrolytes is also provided.

Modeling the Partition Function. Earlier attempts to model liquid started from van der Waals, whose famous equation of state shows the existence of both gaseous and liquid phases. LennardJones and Devonshire [25] summarized other major efforts in developing the equation of states and proposed a lattice model (also called cell model) for liquids. The lattice model assumes each liquid molecule is confined in a cell of certain volume equaling the total volume divided by the number of molecules. They wrote down the partition function for an individual molecule in the cell, including both the kinetic part, which is conventional, and a potential part which depends on the intermolecular potential. The total partition function was obtained from the products of the single particle partition function, from which the Helmoholz free energy is known and the rest of the thermodynamic properties, including the equation of state, can be obtained. Later, Lennard-Jones and Devonshire [26,27] modified their lattice theory to include the free-volume idea of Eyring and Hirschfelder [28] to model latent heats of evaporation and melting.

Eyring and co-workers pictured the existences of empty space in liquids called holes whose size is of the order of a single molecule $[28,29]$. They argued that the energy it takes to create a hole equals the energy needed to evaporate a molecule. They also developed the idea of "free volume," a region surrounding each molecule. Although the free-volume concept had been used by various researchers, its definition was loose and different researchers used different definitions [26,28,30]. In fact, Eyring defined free volume based on the partition function. Models for free volume had been developed, for example, as the average volume that a molecule occupies in the liquid after subtracting the size of the molecule, which can be calculated from its density and the molecular diameter of the molecule. These models allowed the calculation of the partition function. In liquids, Eyring's theory assumes that the total free volume is available to all molecules, as in gases. The sharing of the free volume increases the entropy of molecules, which they called communal entropy. Eyring's research along this 


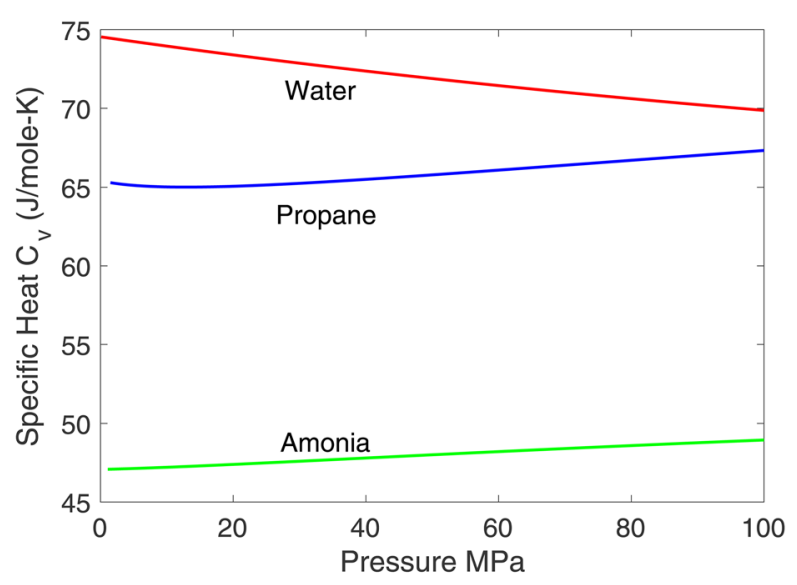

(a)

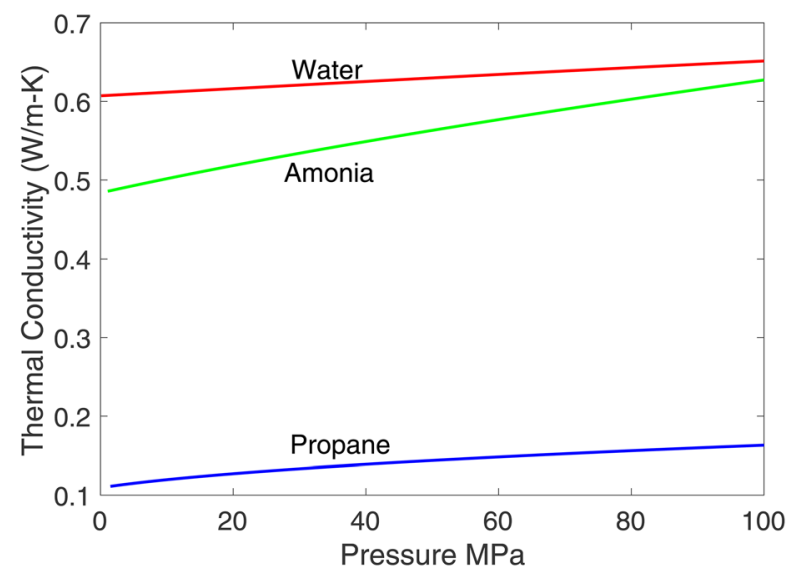

(b)

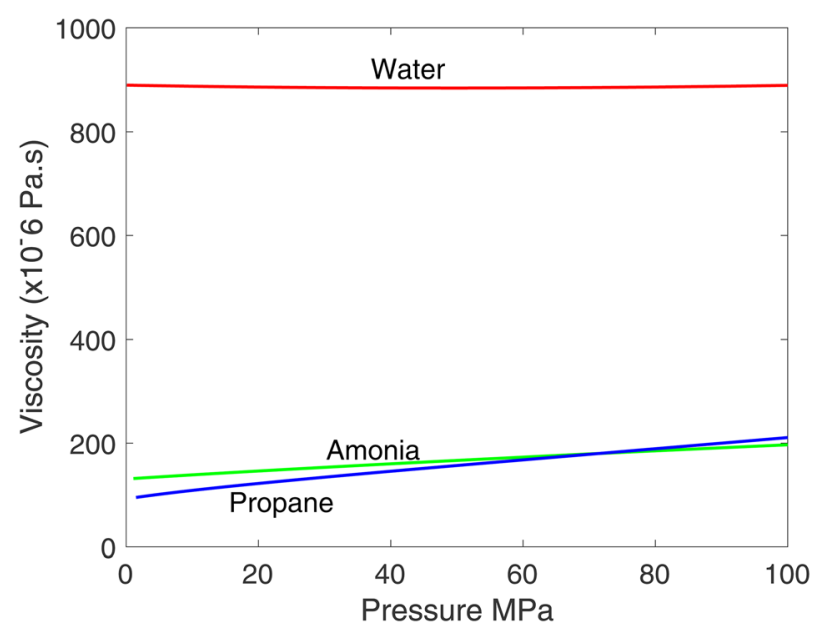

(c)

Fig. 5 Dependences of (a) specific heat, (b) thermal conductivity, and (c) viscosity on pressure at $25^{\circ} \mathrm{C}$

line evolved into the "significant structure theory" [31]. In this theory, holes in liquid are treated as they are moving in the vapor phase, like the well-developed idea of "holes" in semiconductors. The liquid portion is treated as a solid lattice. From this picture, the partition function is the product of that of the holes in the gas phase and that of the liquid in the solid phase. From the partition function, other thermodynamic properties can be obtained.

The intuitive lattice, hole, and free-volume ideas and models had some success in describing thermodynamic properties, including the revision of Gibbs entropy of mixing expression to polymer solutions to account for the large volume difference between the polymer and the solvents by Flory [32] and Hildebrand [33]. Such modeling approaches, however, had gradually fallen out of favor [24] and yielded to more rigorous statistical thermodynamics treatments.

Rigorous Statistical Formulation. One line of research to formulate a theory on liquid is to start from the Liouville equation, which is exact in describing the time-evolution of the $N$-particle distribution function in the phase space of the particles' positions and momenta, and progressively reduces the distribution functions to lower orders, the $n$-particle distribution function, by integrating over the phase-space variables of the rest $(N-n)$ particles [11]. This reduction method is called BBGKY hierarchy in honor of the key contributors Bogoliubov-Born-Green-Kirkwood-Yvon [34-37]. The Boltzmann transport equation can be derived from this hierarchy when the $N$-particle distribution function is reduced to the one-particle distribution function assuming collision between molecules destroys their trajectory memory (molecular chao assumption) and negligible molecular size. The $n$-particle distribution function can be expressed as the product of an explicit term related to the momenta of the particles and an $n$-particle density function $\rho^{(n)}\left(\mathbf{r}_{1}, \ldots, \mathbf{r}_{n}\right)$ related to the potential energy and the $n$-particles' spatial coordinates. At equilibrium, the normalized two-particle density function $g^{(2)}\left(\mathbf{r}_{1}, \mathbf{r}_{2}\right)=\rho^{(2)}\left(\mathbf{r}_{1}, \mathbf{r}_{2}\right) / \rho^{2}(\rho$ is the density) is the pair-distribution function, also called the radial distribution function if the fluid is isotropic so that $g^{(2)}(r)$ is a function of radius $r=\left|\mathbf{r}_{1}-\mathbf{r}_{2}\right|$ only. Since $g^{(2)}\left(\mathbf{r}_{1}, \mathbf{r}_{2}\right)$ approaches 1 as the two particles are separated further and further, another function $h^{(2)}\left(\mathbf{r}_{1}, \mathbf{r}_{2}\right)=g^{(2)}-1$ is defined as the pair correlation function, which can be further related to the direct correlation function $c^{(2)}\left(\mathbf{r}_{1}, \quad \mathbf{r}_{2}\right)$ between a pair of molecules through the Ornstein-Zernike equation [11,38]. The Ornstein-Zernike equation is an integral equation containing both $h^{(2)}$ and $c^{(2)}$ and its closure needs modeling. One often used model was due to Percus and Yevick [39], leading to the Percus-Yevick integral equation relating the radial distribution function $g^{(2)}(r)$ to the intermolecular potential. Once the radial distribution function is known, other thermodynamic properties can be calculated.

For transport properties, Enskog [9] modified the Boltzmanntransport-equation-based approach for liquids. Rather than the one-particle distribution, he modeled the two-particular distribution function as a product of the one-particle distribution function and the radial distribution function. Using a hard sphere model for the latter, he obtained transport coefficients such as thermal conductivity and viscosity. Although this approach has limited success on dense gases, it forms a basis that many subsequent theories compared against.

Another line of attack started from Einstein [10] in his study of the Brownian motion. Einstein's goal was to determine the molecular diameter $d$. Einstein's approach was generalized by Langevin into the Langevin equation [40], which splits the force acting on a Brownian particle by a frictional force that is proportional to the velocity of the particle with a friction coefficient and a random force acting on the particle. Around the same time, Smoluchowski [41] developed a deterministic partial differential equation approach for the evolution of the probability distribution function of a particle doing random walk, which was extended by Fokker and Planck into Fokker-Planck equation (see Chandrasekhar's review [42] and his extension of the Fokker-Planck equation). It has been shown that the stochastic Langevin equation and the deterministic Fokker-Planck equation are equivalent. Landau and Lifshitz developed the fluctuating hydrodynamics approach by adding a fluctuating term to the stress in the Navier-Stokes equations $[43,44]$. Professor Ayyaswamy's group studied extensively $[45,46]$ the Brownian motion of nanoparticles, including the effect of the Brownian particle shape and geometries [47-49], using fluctuating hydrodynamics [50], deterministic approach [51], and 
a hybrid approach they invented [52] combining both fluctuating hydrodynamics and generalized Langevin equation.

Both the Langevin equation and the Fokker-Planck equation include the friction coefficient experienced by the Brownian particle as the input parameter. Along the BBGKY hierarchy, Born and Green [35,53] and especially Kirkwood [36] derived equations for one and two-particle distribution functions. Kirkwood's treatment lies in his approximation to $n=3$, which he assumed to be dependent on the products of the two-particle distribution function. Equations derived by Kirkwood for the distribution functions, in addition to terms similar to that in the Boltzmann transport equation also include a frictional term as in the Fokker-Planck equation. Kirkwood's formulation allows one to calculate the friction coefficient based on the intermolecular potential. Irving and Kirkwood [54] derived expressions for transport coefficients including viscosity, atomic diffusivity, and thermal conductivity. Along the way, they related the heat and momentum fluxes to atomic positions, velocities, and interatomic potentials through derivation of hydrodynamic transport equations (Navier-Stokes equations) from atomic positions and velocities. They showed that the kinetic contribution to the heat flux is related to the one-particle distribution function, while the potential energy contribution is related to the two-particle distribution function.

Kirkwood and his collaborators extensively studied transport properties of liquids by solving for the distribution functions from the equations they developed, some of which we mention later. Overall, however, the success is limited, probably because of the following reasons. One of the key assumptions in the Kirkwood theory [36] is that the frictional coefficient representing the average frictional force on a molecule approaches a constant between the time interval between the random force consecutively acting on the molecule. Alder and Wainwright' molecular dynamics simulation [55], however, showed that the velocity correlation function's decay with time follows a much slower power law than the exponential decay as predicted based on the assumption that the frictional force is proportional to velocity. This was recognized as due to the transient effect when the Brownian particle is kicked by the random force. A generalized Langevin equation that includes memory effect had been developed [48,56]. Interestingly, the Einstein relation still holds true [57]. Kirkwood also commented that the frictional coefficient approaching a constant is not a valid idea in a harmonic crystal, although he surmised that anharmonic interactions would make the approximation valid. Although liquid is certainly not a harmonic crystal, the existence of phonon modes we discuss later leads to further questions on the validity of the Kirkwood's theory.

Monte Carlo and Molecular Dynamics Simulations. Rather than modeling or statistical treatments discussed above, molecular dynamics or Monte Carlo simulations of the entire $N$-particle system are increasingly preferred in studying thermophysical properties of liquids. The Monte Carlo method is faster and more suitable for thermodynamic properties, while simulation of transport properties relies more on molecular dynamics simulations.

Classical molecular dynamics simulation evolves the system in time using Newtonian mechanics or its variants (Lagrangian or Hamiltonian equations of motion) and model intermolecular potentials [2]. Quantum molecular dynamics simulation evolves the system based on the Schrodinger equation or the Dirac equations (for relativistic effect). A popular quantum simulation approach is the Car-Parrinello [58] scheme in which the molecular motion follows classical equations of motion, but the force is computed from solving the Schrodinger equation or the Dirac equations.

Molecular dynamics simulations can be divided into nonequilibrium and equilibrium methods. Nonequilibrium methods mimic real transport by imposing velocity or temperature gradients, but are prone to artificial boundary effects, which can be alleviated by increasing the simulation domain size. Equilibrium methods are based on the linear-response theory, which starts from the Liouville equation and arrives at a formal solution of the perturbed $\mathrm{N}$-particle distribution function subject to an external or internal disturbance under linearization approximation [2]. Such perturbations create response of the system. The response function, or susceptibility in the frequency domain, can be calculated from the Green-Kubo formalism, using the correlation functions of the phase-space variables of the system $[59,60]$. These phase-space variables can be calculated using molecular dynamics simulation results. Expressions for macroscopy quantities of interests such as heat flux, shear stress in terms of the phase-space variables had been derived following the pioneering work of Irving and Kirkwood in liquids [11,54]. In studying solid, Hardy [61] derived a general expression for heat flux that also applies to liquids, which is identical to that of Irving and Kirkwood.

Monte Carlo simulations evolve the system also according to the equation of motion. After a number of simulation steps, the Monte Carlo method decides whether to take the system configuration as one of its samples based on the probability density that the system is supposed to follow: canonical ensemble, grand canonical ensemble, etc. The accepted system forms an ensemble that obeys the prescribed statistical distribution. The thermodynamic properties of the system can be calculated based on the ensemble average of the phase-space expression for the desired properties. Ungerer et al. [62] summarized Monte Carlo and molecular dynamics-based simulation of thermophysical properties of liquids. We will discuss some insights learnt from these simulations later.

Phonon Theories. Based on the previous discussion, we see that most treatments of liquids try to modify their departure from gases, because liquids flow as gases. However, the densities of liquids are close to solids. Frenkel maintained that liquid is close to solid and introduced the phonon theory $[12,63,64]$. In his theory, the liquid oscillates largely around its equilibrium positions but occasionally jumps from one location to another with a characteristic time $\tau$. If the applied external shear force has a characteristic time longer than $\tau$, then many such jumps happen and liquid moves as we normally understand it. However, if the external shear is faster than $\tau$, the liquid behaves as solid and can sustain the shear stress. Frenkel's theory has some root in Maxwell who had pictured that liquid stress consists of an elastic part like a rigid body and a viscous part of a liquid body [13]. Frenkel further identified that this relaxation time is approximately the Maxwell relaxation time for viscoelastic bodies

$$
\tau \approx \tau_{M}=\frac{\mu}{G}
$$

where $\mu$ is viscosity, and $G$ is the shear modulus of the liquid at high frequency. Frenkel explained that the jumping of the molecules is due to the hole formation, consistent with Eyring's picture.

Although Frenkel's phonon theory had been sidelined in favor of statistical approaches championed by Kirkwood and others, it gained new momentum led by Trachenko and Brazhkin [14], who cited the experimental observation of high-frequency transverse phonons $[65,66]$ in liquids as strong evidence in support of the phonon theory of liquid. The phonon theory also resembles Eyring's significant structure theory [31], although the latter treated the "solid part" of liquid with an Einstein harmonic-oscillator model, while recent treatment of Trachenko used the Debye model. So far, the phonon model has mostly dealt with intermolecular modes only and neglected intramolecular modes. A treatment by Prigogine et al. [67] on the partition function of liquid polymers considered both intra and intermolecular vibrational modes, although it eventually neglected the intramolecular modes.

Two other pictures are also largely consistent with the phonon theory: the instantaneous normal-mode theory and the two-phase thermodynamic model. 
The instantaneous normal-mode theory $[68,69]$ approximates the liquid at short time scale as harmonic vibrational and rotational modes and uses lattice dynamics method as in the solidstate treatment to find the eigenfrequencies of these instantaneous normal modes. One distinct feature of the instantaneous normal modes is the existence of imaginary frequencies, which in real space means instability of the lattice: the amplitude of some of these modes can grow exponentially and molecules can move to other positions. Another distinct feature is that the density of states at zero frequency is not zero. Analysis of instantaneous normal modes in water shows that lower frequency modes are extended rather than localized [70]. Self-diffusivity of liquid is related to the spectrum of the density of states, although the validity of this relation was questioned $[68,71,72]$.

The two-phase thermodynamic model developed by Goddard and co-workers bears similarities to the phonon model and instantaneous normal-mode analysis $[73,74]$. Instead of harmonic analysis and the corresponding density of states, molecular dynamics simulations are used to extract the density of states of the modes from the velocity autocorrelation function. Compared to the density of states obtained from the instantaneous normal-mode analysis, such an analysis does not lead to any information on the imaginary frequency part, although such information was not used in the instantaneous normal analysis neither. Like the instantaneous normal-mode analysis, the density of states at zero frequency is not zero. Lin et al. [73] related the zero-frequency density of states to the atomic diffusion coefficient. They further argued that the density of states consists of two parts, one is the gas-like part, for which the density of states has analytical expression, and a solid-like part. They developed models to determine the fraction of gas-like and solid-like modes. This two-part decomposition was also extended to include rotational motion in liquids. These approaches enable one to calculate thermodynamic properties such as entropy and specific heat of liquids. This two-part picture is also consistent with Eyring's significant structure theory [31].

Iwashita et al. [75] carried out both classical and quantum ab initio molecular dynamics simulation on the Maxwell relaxation time on metals and compared it with the lifetime of local atomic connectivity $\tau_{\mathrm{LC}}$, defined as the time for an atom to lose or gain one nearest neighbor. They found that $\tau_{M}=\tau_{\mathrm{LC}}$ for temperature above certain critical temperature. They reasoned that rather than phonon picture, local atomic connectivity is a more appropriate excitation mode in interpreting the viscosity.

Water and Other Polar Liquids and Electrolytes. Water, together with air, is the most used heat transfer fluid. Despite its prevalence in our daily life, water is considered the most complicated fluid. Due to the hydrogen bonds that can extend a long range, many puzzles of water continue to attract extensive studies [76,77], such as the density maximum at $4{ }^{\circ} \mathrm{C}$ and smaller density of ice. Water is considered to have anomalously high melting and boiling points and critical temperature compared to other lowmolecular weight substances. The $\mathrm{OH}$ bonds in water are highly polar and directional. The polar field of $\mathrm{OH}$ bond distorts electrons in oxygen atom of a neighboring water molecule, forming the hydrogen bond. In common hexagonal ice crystal structure [76], a water molecule is surrounded by four hydrogen bonds. Using the latent heat of evaporation of water $2.26 \mathrm{MJ} / \mathrm{kg}$, we can estimate the average energy to break off a water molecule is $\sim 0.21 \mathrm{eV}$.

In water, the average hydrogen bonds per molecule are between 2.3 and 3.6 according to molecular dynamics simulations, depending on the potential used [76,77]. This number is lower than 4 in ice and suggests that some hydrogen bonds are broken in water. A few prevailing models of water picture hydrogen-bonded clusters forming and breaking as reviewed by Ludwig [77]. Benson and Siebert [78] modeled water that consists of dynamic equilibrium between cubic-shaped octamers which dissociate into two cyclic tetramers. Chaplin [79] proposed that water consists of a fluctuating hydrogen-bond network of icosahedral symmetry with $280 \mathrm{H}_{2} \mathrm{O}$ molecules, consisting of hexamer and pentamer rings as subunits. Weinhold [80] pictured water as an equilibrium among many different flickering clusters, whose properties can be calculated based on ab initio method, from which the partition functions can be constructed and used to calculate thermodynamic properties-the quantum cluster equilibrium theory. Although none of these theories can completely reproduce all properties of water, it is an accepted picture that water consists of hydrogenbonded clusters in dynamic equilibrium. Water is an extreme of hydrogen-bond dominated liquids; other polar liquids, such as ammonia and alcohol, also have hydrogen bonds.

Electrolytes contain dissolved ions. Due to their strong electrical field, $\sim 10^{6} \mathrm{~V} / \mathrm{cm}$ around a radius of $2-3 \AA$, ion addition into water significantly disturbs the water structure. Around a single ion, the effective dielectric constant is much lower because the high field of the ion restrains the rotation of water molecules. Frank and Wen [81] suggested that water surrounding an ion is divided into three regions. Region $A$, immediately around an ion, is structure-making, composed of immobilized water molecules due to the strong electric field of the ions. Region $B$ is structurebreaking. This intermediate region of water is more random than bulk water. Further away, region $C$ is the bulk water. The more random intermediate region $B$ is due to the competition between normal oriented hydrogen bonds between water molecules and the more spherical electrical field of an ion. The competition between regions $A$ and $B$ makes an ion as either a structure maker (smaller ions and multivalent ions) or structure breaker (larger ions).

Around ions are also counterions in an electrolyte solution. The Debye-Huckel theory based on solving linearized PoissonBoltzmann equation is considered as foundational for understanding many effects of ions [82]. However, the Debye-Huckel theory treated water as a mean field and is only valid in very dilute electrolyte solutions $[83,84]$.

\section{Specific Heat of Liquids}

For solids at high temperatures, the specific heat per mole of atoms is $3 k_{B} N_{A}=3 R$, where $N_{A}$ is the Avogadro constant, and $R$ is the universal gas constant. This Dulong-Petit limit is because each atom has three kinetic energy component $m v_{x}{ }^{2} / 2$ and three potential energy component $k x^{2} / 2$. At high temperatures when the energy equipartition is valid, each degree-of-freedom in energy contributes on average a $k_{B} T / 2$ to the internal energy, leading to $3 k_{B} N_{A} T$ total energy per mole and a specific heat $3 R$. In comparison, the volumetric specific heat of a monatomic gas is $3 R / 2$, because each atom has only the kinetic energy components, no potential energy. The specific heat of diatomic gas increases to $5 R / 2$ per mole of molecules, because of the two additional rotational degrees-of-freedom. At higher temperatures, vibrational modes are excited and specific heat can reach $7 R / 2$.

For simple liquids, the specific heat usually decreases from around $3 R$ at the melting point to $2 R$ at higher temperatures. Brillouin [85] first recognized that during transition from solid to liquid, the two shear modes disappear, so are their contributions to the potential energy $k_{B} N_{A} T$, leading to a specific heat $2 R$. Brillouin also recognized that some of the shear modes can still exist at high frequencies.

Wallace [86] developed a model treating molecules in liquid as if they are sitting in a nearly harmonic potential. He started with a specific heat of $3 R$ under equipartition theory. He then corrected the fact that when a molecule's motion amplitude is larger than certain value, it no longer returns to its equilibrium position. He called this correction as the boundary correction where the boundary is between two adjacent molecules, which he further related to the melting temperature. He also considered the anharmonic vibration contribution to the specific heat and approximated it the same as that of the solid near the melting temperature. His treatment allowed the liquid specific heat to be plotted as a function of temperature to the melting point. 
Trachenko and co-workers $[64,87,88]$ used Frenkel's theory to model the specific heat of liquids. In their model, the energy of a liquid consists of translational energy of the molecules across all frequency range, the potential energy for all the longitudinal vibration modes, the potential energy for the transverse vibrational modes with frequency above the Frenkel frequency, and a potential energy accounting for the diffusion of molecules. They further argued that the latter component is small and can be neglected. Thus, the total energy consists of the longitudinal phonons across the entire frequency spectrum (kinetic and potential) and two branches of transverse phonons with frequency higher than the Frankel frequency and their kinetic energy below the Frankel frequency. They employed a Debye model for the density of states for both longitudinal and transverse phonons and used the Helmholz free energy of phonons to arrive at the internal energy of these phonons. This treatment also allowed them to account for the change of phonon frequency with temperature by modeling it with the Gruneisen parameter and the thermal expansion coefficient. They also applied the phonon model to account for the kinetic energy associated with transverse phonons below that of the Frenkel frequency, despite the fact that the physical picture does not include such phonons. Their model leads to a simple way to calculate the liquid specific heat as a function of temperature, using viscosity, shear modulus, and thermal expansion coefficients as inputs, and the Debye frequency as a fitting parameter. The results obtained are in good agreement in comparison to experimental data on simple liquids after subtracting out rotational contributions. They also reached good agreement for water by assuming the configurational change between water clusters account for half of the $C_{V}$. Recently, Lilley et al. [89] used such a phonon picture to calculate the entropy of liquid and latent heat change of melting and evaporation to explain the Richard's rule for melting and Trouton's rule for evaporation. Their model assumed the existence of transverse and longitudinal phonons at the melting point with frequency between Debye frequency $\omega_{D}$ and $\omega_{D} / \sqrt{2}$, the latter as a cut off of frequency.

Pascal et al. [90] applied the two-phase model championed by Lin et al. $[73,74]$ to study the heat capacity of 15 organic liquids. As mentioned in Sec. 3, the two-phase model splits the density of states obtained from the Fourier transform of the velocity autocorrelation function into a gas-like part and a solid-like part, and models each part using established partition functions for gas and phonons. In their approach, the fractions of gas-like versus solid-like contributions are modeled through a fluidity parameter.

Trachenko's model so far has been on simple liquids, which show decreasing specific heat with increasing temperature. However, Fig. 1 also shows that specific heat for more complicated liquids can increase with temperature. I believe that the reason for this increase is the increasing contributions of internal vibrational modes of the molecules. In simple molecules such as water, the intramolecular vibration modes are at high frequencies and normally are not excited, and hence can be neglected, as Trachenko and his co-workers assumed. However, with increasing molecular size, internal vibration frequency spreads to lower values. Some of the intramolecular vibrational modes can be thermally excited, and the numbers of these modes increase with increasing temperature. One can understand this by constructing a finite onedimensional periodic lattice, starting from a diatomic molecule, which has only one vibration frequency. As more and more atoms are added to the lattice, the acoustic branches form with longer wavelength and lower frequency. Some of these modes can be thermally excited and contribute to the specific heat. This contribution should increase with temperature as more and more internal vibrational modes can be excited with increasing temperature. Such vibrational contributions had been mostly neglected in thermodynamic analysis of liquids. For example, both Prigogine et al. [67] and Flory et al. [91] neglected the intramolecular contributions to the partition function. Interestingly, Prigogine and coworkers had used internal coordinates to decompose the internal vibration into normal modes, although they eventually neglected these modes.

In Fig. 1, the specific heat was plotted using per mole of molecules. In Fig. 6, we replot $C_{v}$ into $\left(C_{v} / R-1.5\right) / N$, where $N$ is the number of atoms in the molecule. $C_{v} / R-1.5$ assumes that each molecule's kinetic energy mode is fully excited, and they contribute $3 R / 2$ (per mole of molecule) to $C_{v}$. This contribution is subtracted out in Fig. 6. First, we note that nitrogen, ammonia, and $\mathrm{CO}_{2}$ values fall between 1 and 1.5 and decrease with increasing temperature $\left(\mathrm{CO}_{2}\right.$ has a minimum), which could be due to rotational mode contributions (fully excited rotational mode contributes $1 R$ for a linear molecule such as nitrogen) or due to potential energy contributions. We note that on the atom molar fraction basis, the specific heat of dodecane is higher than propane, which is higher than ethane, because more and more internal vibrational modes can be excited as frequency shift downwards, which also explains the reversal of temperature dependence of the specific heat from ethane to propane and dodecane.

In a review paper, Ungerer et al. [62] noticed that one interesting study reported that the excess specific heat (above that of ideal gas) shows a peak with pressure for methane. This was interpreted as due to the simulated methane was above the critical point. This is consistent with the picture that in the vapor phase above the critical point, the potential energy contribution to the specific heat diminishes, as in Trachenko's theory.

The phonon model of Trachenko and co-workers and the twophase model of Goddard and co-workers share some common physical pictures that are also consistent with the instantaneous normal-mode theory and resemble Eyring's significant structure theory. The two-phase model splits the modes into a gas-like part and a solid-like part and used the fluidity parameter to model the fraction of gas-like and solid-like modes. Trachenko and coworkers employed Frenkel frequency to split the gas-like and solid-like modes, which avoid the fluidity parameter needed in the two-phase model. However, Trachenko also used the same phonon model to treat the kinetic energy contribution below the Frenkel frequency, which is questionable since these are not vibrational modes. Rather, it might be more reasonable to assume each of these kinetic modes contributes $k_{B} T / 2$ as they are fully excited. Although this might be a more correct physical picture, I expect that the numerical difference will be small as long as the Frenkel frequency is low, because in this case the vibrational modes as used in the Trachenko model are fully excited, leading to $k_{B} T / 2$ per mode contribution to the internal energy. The twophase model requires molecular dynamics simulation. In comparison, the instantaneous normal-mode theory treats all real frequency of the instantaneous modes as vibrational modes and imaginary frequency for the gas-like mode. The zero-frequency density of states was used to obtain the self-diffusion constant.

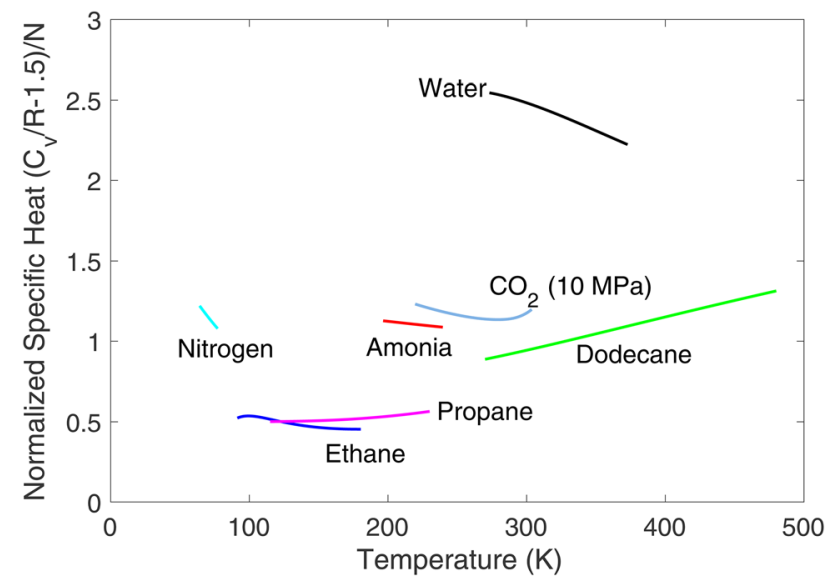

Fig. 6 Normalized specific heat of selected liquids to number of atoms in a molecule $N$ 
Given the success of the Trachenko phonon model, it will be interesting to check if the instantaneous normal-mode theory can be expanded to predict specific heat.

\section{Thermal Conductivity of Liquids: Modeling and Simulation Approaches}

Thermal conductivity is difficult to measure and to predict. The review by McLaughlin [92] is probably still the most comprehensive one despite the fact it was published in 1964. Touloukian and Ho [93] also gave a good summary of previous theoretical work as well as empirical correlations, in addition to extensive data on thermal conductivity. In this section, we will discuss a few models on thermal conductivity of liquids and group them into (1) kinetic models, (2) phonon models, and (3) molecular dynamics simulation. In Sec. 6, we will discuss thermal conductivity of representative liquids.

Kinetic Models. Many researchers started from modifying the kinetic transport picture of Boltzmann for dilute gas particles for transport in liquids, as represented by Enskog's approach [2,9,94]. Solution of the Enskog equation leads to a thermal conductivity expression that includes convective and collision contributions, compared to that of a dilute gas consisting of only convective contributions. Enskog further splits the convective term into intracellular contribution and intercellular contribution but concluded that both of their contributions are small. Enskog's theory has some success for gas phase at high density but does not work for liquid.

Bridgman constructed a model to explain the thermal conductivity that he measured [95]. He imagined that the liquid molecules sit on a cubic lattice and assumed that the internal energy of a molecule is $2 k_{B} T$, so that the energy transferred between adjacent molecules is $2 k_{B} \delta d T / d x$, where $\delta$ is the distance between two adjacent molecules, which can be calculated based on the molecule's mass and density $\delta=(\mathrm{m} / \rho)^{1 / 3}$. He further assumed that the frequency of this energy transfer happens at $v / \delta$ where $v$ is the velocity of sound. The total number of lattice point per unit area is $1 / \delta^{3}$, which leads to the following expression for thermal conductivity:

$$
k=2 k_{B} v / \delta^{2}=2 k_{B} v\left(\frac{m}{\rho}\right)^{-2 / 3}
$$

He stated that this formula gives right temperature dependence trend not only for regular liquids that show thermal conductivity decreases with increasing temperature but also for water that has opposite trends. The predicted pressure dependence, however, is greater than the actual case. Similarly, Sakiadis and Coates used a classical kinetic model for thermal conductivity $k=\rho C_{p} v \delta[96,97]$ to explain their measurements on thermal conductivity and specific heat of $\sim 50$ organic liquids.

Horrocks and McLaughlin [98] started from the liquid lattice picture of Lennard-Jones and Devonshire [25] and considered the energy exchange between successive layers due to potential energy exchange and molecular hopping, analogous to a simple derivation of the Fourier law from kinetic theory [2]. They arrived at the following expression for the contribution of potential energy exchange between two adjacent molecular layers to the thermal conductivity $k=2 n P \nu \Lambda c_{v}$, where $c_{v}$ is the specific heat of a molecule, $P$ is the probability that energy is transferred when two vibrating molecules collide, $n$ is the number of molecules per unit area of the liquid quasi-lattice, $\nu$ is the intermolecular vibration frequency, and $\Lambda$ is the distance between successive molecular layers. They used van der Waals potential to derive a characteristic vibrational frequency, as in the Einstein model. They also evaluated contribution due to molecules jumping between atomic planes based on the free-volume concept, i.e., the "convection" term. Their analysis showed that the contribution of the convection term is small. They took $P=1$ and $c_{v}=3 k_{B}$ in estimating the vibrational contribution to thermal conductivity.

If we replace $C_{p}$ with $2 R$, the Sakiadis and Coates expression becomes identical to that of the Bridgman formula. We should keep in mind, however, that the correct specific heat to use for thermal conductivity is $C_{v}$ rather than $C_{p}$. If one takes $\delta \nu$ in the Horrocks and McLaughlin model as the speed of sound $v$, their formula also becomes similar to the Bridgman formula since $n=1 / \delta^{2}$, except that Horrocks and McLaughlin have an extra factor of 2 and used a specific heat of $3 k_{B}$ per molecule. More recently, Xi et al. [99] extended this line of modeling and developed athermal conductivity expression, which they claimed is valid for both liquids and amorphous solids.

Longuet-Higgins and Pople developed a model aiming to compute the rate of momentum exchange during two-particle collision in a similar way to the kinetic theory, using a hard sphere model with infinite potential [100]. However, their predicted thermal conductivity increases with $T^{1 / 2}$, as in gases, contrary to observation in most liquids' thermal conductivity. To relax the infinite potential approximation, Longuet-Higgins and Valleau [101] further considered a square potential well. Yet the results still cannot well capture the temperature dependence of the Prandtl number.

Another line of attack is based on the Kirkwood theory, with main differences among different researchers on how to approximate the interatomic potentials [102,103]. None of these approaches, however, can lead to correct dependence of thermal conductivity on temperature compared to experimental data. The best agreement along the Kirkwood theory was the work of Rice and Allnatt [104], which assumed a hard sphere repulsive potential and a soft attractive potential. Rice and co-workers [105,106] further refined the theory and conducted measurements on thermal conductivity and self-diffusion in simple liquid like $\mathrm{Ar}, \mathrm{Kr}, \mathrm{Xe}$, and $\mathrm{CH}_{4}$ to compare theory and experiments. They found good agreements on thermal conductivity when an experimental friction constant was used. However, the same theory does not lead to good agreement with the diffusion coefficient.

Rather than the Kirkwood approach which connects with the Langevin equation via the friction coefficient, Eisenschitz used the modified Smoluchowski equation [107], which is a special case of the Fokker-Planck equation, and derived expression for the two-particle distribution function and thermal conductivity. His expression depends on the frictional coefficient in the Smoluchoswki equation and hence cannot be independently evaluated, although his theory predicted different trends of thermal conductivity and viscosity dependence on temperature.

Phonon Model. In some sense, the previously discussed lattice-based models by Bridgman [95] and Horrocks and McLaughlin [98] can be thought of as phonon-based, although we placed them in a different category. Based on the significant structure theory, Eyring and co-workers [108] argued that the thermal conductivity of liquid consists of two parts, one is due to holes that can be modeled as gas and the rest of liquid part can be treated as a solid with lattice vibration, for which they used the Callaway model for solids [109], including Klemens expressions [110] for the relaxation time to fit the experimental data.

Eyring's model did not make distinctions for molecular motion slower than the Frenkel frequency. Zhao et al. [111] combined Trachenko's idea for specific heat with the kinetic theory for thermal conductivity to model thermal conductivity of liquids. They started from the standard kinetic theory for the spectral expression of phonon thermal conductivity [2], splitting them into longitudinal and transverse branches. For the latter, they used the Frenkel frequency as the lower cutoff frequency. They then invoked the gray model approximation: all phonons in liquid have same group velocity equaling the sound velocity, and they took the interatomic distance as the phonon mean free path. Their approximations lead to the standard kinetic expression for thermal conductivity $k=C_{p} v \delta / 3$, similar to that of Bridgman, and 
Horrocks and McLaughlin, except the difference in the prefactor. Their calculation shows that the model is reasonable for liquids of low molecule weight but fails for liquids of large molecule weight.

Trachenko et al. [112] provided fundamental insights on the transition between liquid and gas phases via investigating speed of sound, viscosity, and thermal conductivity, based on considering the continuous transition between liquid and vapor states above the supercritical states. In the liquid state, thermal conductivity decreases with temperature due to the decreasing modes of transverse phonons as in the specific heat. But in the vapor state, thermal conductivity increases with temperature because of the increasing mean free path and velocity with temperature, as is predicted from the kinetic theory. This means a minimal should exist, which they did see based on existing experimental data. Prior work on casting thermal conductivity in reduced state properties also showed a minimum in thermal conductivity but no insights were provided [113]. Trachenko and co-workers then started from the kinetic expression and argued that the fundamental limit is the UV cut off, i.e., the minimum wavelength is limited by the atomic size. They made similar arguments for viscosity and speed of sound $[114,115]$. Through scaling of the interatomic distance to the Bohr radius and interatomic energy to the Ryderberg energy, they related the minimum values of thermal diffusivity and viscosity to fundamental physical constants

$$
D=\nu=\frac{1}{4 \pi} \frac{\hbar}{\sqrt{m_{e} m}}
$$

where $\hbar$ is the reduced Planck constant, $m_{e}$ is the electron mass, and $m$ is the molecule mass.

Molecular Dynamics Simulations. Molecular dynamics simulations have been increasingly used in simulating thermal conductivity, especially in amorphous materials that cannot be dealt with by first-principles simulations [116-119]. Ohara and co-workers carried out extensive molecular dynamics simulations on a variety of liquids and developed decomposition techniques to gain insights on heat conduction mechanisms in liquids. Most of their studies used nonequilibrium molecular dynamics to obtain thermal conductivity, but they demonstrated the equivalence between equilibrium and nonequilibrium decomposition methods [120]. They extended the heat flux expression to include multibody potentials [121] and pointed out delicacy in different ways to calculate heat flux [122]. For liquids described by a Lennard-Jones potential, the simulation suggests around $90 \%$ of the heat flow is due to intermolecular potential rather than convective motion of the molecules [123], consistent with many previous modelingbased studies we mentioned.

\section{Thermal Conductivity of Selected Liquids}

This section will discuss experimental trends of thermal conductivity and heat conduction mechanisms in representative liquids.

Organics Liquids. Measurements of the thermal conductivity dependence on the number of carbon atoms $n$ in alkenes seem to suggest that thermal conductivity increases with $n$ almost linearly between 22 and 32 [124] but decreases with increasing temperature. Experimentally, the measured specific heat does not change much with $n$ and saturates after $n=10$ [125] and usually increases with increasing temperature. The increasing thermal conductivity with $n$ could be explained by the increasing volume occupied by each molecule, which has a high thermal conductivity [126]. The decreasing thermal conductivity with increasing temperature might be due to two reasons: (1) increasing intermolecular spacing due to volume expansion, and (2) increasing scattering of intramolecular phonons by intermolecular force, which reduces the intrachain heat conduction. The first mechanism is common to most liquids, while the second one could be important for long chain molecules. Whether one or both of these two pictures is responsible for decreasing thermal conductivity with increasing temperature remains to be explored.

Ohara and co-workers used molecular dynamics simulation to simulate thermal conductivity of organic liquids $[127,128]$. They found that in $n$-octane, $\sim 75 \%$ of the heat is conducted due to potential energy interactions, while rest is by convective contributions. Around $37 \%$ of the $75 \%$ potential interaction is due to intramolecular interactions. For $n$-alkanes, the intramolecular contributions increase with chain length, while the intermolecular contributions decrease. It was observed from the simulation that the thermal conductivity depends on the main chain length, while the side chain location and lengths have negligible effects. Since the simulation temperatures varied between different molecules, it was difficult to extract a trend of the dependence of thermal conductivity on carbon atoms along the chain to compare with the experimentally observed trend. They also studied thermal conductivity of alcohol of different chain length [129] and compared thermal conductivity of ethanol and ethylene glycol [130]. They observed that the long range Coulomb interactions make substantial contributions. In alcohol, the Coulomb contribution decreases with increasing chain length due to longer separation of the charged groups. Ethylene glycol has larger thermal conductivity than ethanol because of higher $\mathrm{OH}$ bond density. They observed a shallow minimal of thermal conductivity when there are four carbons along the alcohol chain, consistent with experiments, although they cautioned that experiments might be wrong and suspected thermal conductivity of longer chains might be a constant. However, based on the experimental data on longer alkane chains [124], the thermal conductivity does increase with chain length, likely due to increased intrachain heat conduction.

Yang et al. [131] used nonequilibrium molecular dynamics simulation to study thermal conductivity of liquid aldehydes and ketones with carbon varying between 4 and 10. They found that thermal conductivity of aldehydes first decreases with carbon number and then increases. They observed especially an increase of torsional motion contribution to thermal conductivity. Although they reported kinetic and potential energy contributions, they did not separate them into intermolecular and intramolecular contributions, the latter would be useful for understanding heat conduction mechanisms.

Water and Polar Liquids. Water's thermal conductivity temperature dependence is more difficult to explain. As shown in Figs. 1 and 2, the specific heat of water decreases with increasing temperature, consistent with most other liquids. However, thermal conductivity of water increases with temperature. Some acids (nitric acid $\mathrm{HNO}_{3}$ and sulfuric acid $\mathrm{H}_{2} \mathrm{SO}_{4}$, for example) also show thermal conductivity increases with increasing temperature [17].

Using molecular dynamics simulations, Ohara [132] further divided the intermolecular energy exchange into translational and rotational contributions and concluded the intermolecular rotational mode contributes dominantly to this exchange in water. This conclusion seems to conflict with experiments comparing thermal conductivity of $\mathrm{H}_{2} \mathrm{O}$ and $\mathrm{D}_{2} \mathrm{O}$ [93]. Based on dimensional analysis, the ratios of transport properties $\left(\mu_{1} / \mu, k / k_{1}\right.$, and $\left.D / D_{1}\right)$ (viscosity, thermal conductivity, and diffusivity) should be proportional to the square root of the ratio of mass $\left(m_{1} / m\right)^{1 / 2}$ or moment of inertia $\left(I_{1} / I\right)^{1 / 2}$. When comparing to experiments, it was found that the while viscosity ratio seems to be more correlated to $\left(I_{1} / I\right)^{1 / 2}$, the thermal conductivity ratio is more correlated to the mass ratio $\left(m_{1} / m\right)^{1 / 2}$, suggesting that the rotational motion of molecules does not contribute much to thermal conductivity. At this stage, the reason for the conflict between simulation and experiments is unclear.

In a comparison of thermal conductivity between water and ammonia, Matsubara et al. [128] showed that the contribution from Coulombic contributions in water, either due to hydrogen 
bond or due to nonhydrogen bond, dominates over that due to the van der Waals interaction, with a ratio of $\sim 7: 1$; while in ammonia, the ratio is only $1.5: 1$, despite that the total thermal conductivity of water and ammonia is close. These results are consistent with the picture that the hydrogen bond in water is much stronger than in ammonia [79]. A comparison of the impacts of different potentials of water was presented by Sirk et al. [133].

Clearly, the hydrogen bond has something to do with the experimentally observed trend of thermal conductivity increasing with temperature in water and other polar liquids. Palmer suggested that thermal conductivity increases because at higher temperatures, hydrogen bonds are broken, and at lower temperatures, they recondense [134]. Palmer modified an empirical relation to include the latent heat in the thermal conductivity expression for associated liquids. The validity of this picture could be examined with molecular dynamics simulations.

The range of Coulombic interaction should depend on the dielectric constant via Debye length. So far, there is no exploration of this connection. The dielectric constant of water is around 80 compared to 20 of ammonia, suggesting that Coulombic interaction in water should be much stronger, consistent with Ohara's simulation. However, the competition between van der Waals force and Coulombic force is not well understood. We are far from understanding heat conduction in polar liquids.

Electrolytes. As we discussed before, the solvent structures surrounding ions are complicated, which can impact thermal conductivity. Most electrolytes' thermal conductivity decreases with increasing electrolyte concentration [135]. However, sodium fluoride in water showed a peak in thermal conductivity around 0.5 molal NaF, as shown in Fig. 7 [135].

The ability to increase thermal conductivity beyond pure water with $\mathrm{NaF}$ is interesting, as water is among the most heatconductive liquids other than liquid metals and is certainly the most widely used heat transfer liquid together with air. The activity coefficients between $\mathrm{LiCl}$ and $\mathrm{NaF}$ do not seem to differ much [136], and hence they cannot explain the experimental observation. However, both $\mathrm{Na}^{+}$and $\mathrm{F}^{-}$ions are water structure makers, while $\mathrm{Cl}^{-}$ion is a structure breaker. These facts may explain why the addition of $\mathrm{NaF}$ into water increases its thermal conductivity. Indeed, molar volume of $\mathrm{NaF}$ is negative, while that of $\mathrm{KF}$ is positive [137-139]. The decreasing volume with addition of such ions can lead to decreased intermolecular spacing and stronger force interactions, both of which can lead to increased thermal conductivity. Structure makers usually decrease entropy, while structure breakers increase entropy [140]. Hence, entropy increase cannot explain the thermal conductivity trend. We also notice that the thermal conductivity of $\mathrm{NaF}$-water electrolytes peaks around 0.6 mole fraction of NaF. This effect is difficult to explain, but I

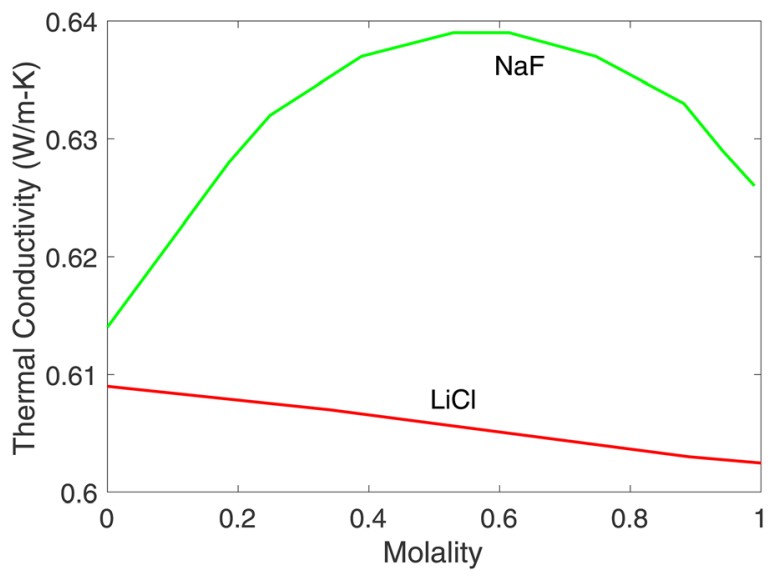

Fig. 7 Thermal conductivity of water as a function of salt ( $\mathrm{LiCl}$ and NaF). Data read from curves in Ref. [135]. suspect that it may be of the same origin of the activity coefficient of many electrolytes first decreases and then increases with increasing mole concentration after reaching a minimum [141]. This effect can be related to the saturation of the surrounding counterions [81,82].

Liu et al. [142] measured transport properties of six different ionic liquids, including specific heat and thermal conductivity, and also carried out molecular dynamics simulations on these transport properties. The experimentally measured thermal conductivity of different liquids showed different temperature dependence. Some increase and some decrease, while others showed nonmonotonic dependence on temperature, but results from their molecular dynamics simulations for all six liquids show that thermal conductivity decreases with increasing temperature. Inaccuracy in intermolecular potential could be the reason.

Liquid Metal. Liquid metals are special for heat transfer due to their much higher thermal conductivity than other liquids. Unlike normal liquids for which the critical temperatures are 1.5-1.75 times their normal boiling temperatures, the critical temperatures of liquid metals are 3-4 times that of their normal boiling points. In liquid metals, electrons are the major heat carriers as in solid metals. Grosse [143] studied electrical and thermal conductivities of liquid metals from melting points to critical points. The electrical conductivity of only a few metals such as mercury had been measured over its entire melting temperature to the critical temperature range, which typically decreases with increasing temperature. Because thermal conductivity measurements of liquid metals are more difficult, reported values between different authors varied widely. Based on the Wiedemann-Franz law, Grosse suggested that for many metals, thermal conductivity increases first with temperature and then decreases, showing a peak in the thermal conductivity.

Mott [144] studied the electrical conductivity mechanisms on liquid metals and developed a model based on the increased amplitude of atomic vibration in the molten state relative to the solid state near the melting point. Ziman [145] argued, however, that the Mott picture, which is based on the Einstein model for liquid, is not correct. Ziman used kinetic picture and considered the impacts of two scattering sources, the "thermal" and "structure," on the electron mean free path. Sobolev and Mirzoev used Kubo-Greenwood formula and linear "muffin-tin" orbital to compute the electrical conductivity of liquid metals [146]. Combining this approach with the Wiedemann-Franz law can lead to estimation of thermal conductivity of metals at high temperatures.

Mixtures. A large amount of experimental data exists for thermal conductivity of mixtures of liquids; some are cited in Ref. [19-21]. In general, thermal conductivities of binary mixtures fall in between the two pure components and change monotonically with composition. Data on $n$-butyl acetate/diethyl ether mixture seem to show a peak at 50/50 weight fraction, but it is unclear if these are experimental errors [21]. Yu et al. [147] measured thermal conductivity of $L$-menthol-decanoic acid deep eutectic solvents as a function of temperature, pressure, and mixture mole concentration for their potential usage in thermal storage. $L$ menthol is a solid at room temperature but forms liquid with decanoic acid in a wide range of composition with different melting temperatures, called deep eutectic. The thermal conductivity behavior of the mixture is regular.

Bearman and co-workers $[148,149]$ studied thermal conductivity of mixtures based on the statistical formulation of Kirkwood [36]. Bearman's results show that thermal conductivity dependence on mole fraction of each species is nonlinear.

Ohara also studied thermal conductivity of binary mixtures of argon and krypton using molecular dynamics simulations [150] and analyzed contributions from convection to potential energy to the transport and contributions from same species and between two species. There are no big surprises in the pictures obtained. 


\section{Viscosity}

Different from thermal conductivity, viscosities of all liquids decrease with increasing temperature as shown in Fig. 3. Andrade [151] developed a kinetic model of viscosity using the following lattice picture. Based on diffusivity data, he first justified that the molecular jump in position is infrequent compared to the frequency of molecular oscillation in its cell. He considered the momentum exchange when a molecule moves to the edge of the cell with the neighboring molecule as the mechanism of momentum exchange between adjacent layers of molecules. His model, however, cannot give the correct temperature dependence of viscosity. Eyring [152] developed a transition state theory for viscosity based on the idea of holes in liquid discussed in Sec. 2 and on energy barriers existing between molecules. His theory requires models of several rate constants and therefore is not straightforward to use.

The most widely used model for viscosity is from Doolittle [153], who had conducted extensive experiments on viscosity and developed a model of viscosity based on the free-volume idea

$$
\mu=A e^{B v_{o} / v_{f}}
$$

where $v_{f}$ is the free volume which he attributes to arising from the total thermal expansion of the liquid, and $v_{o}$ is the volume of the liquid extrapolated to zero temperature without change of phase. Williams et al. [154] further related the free volume and temperature by referencing the volume at the glass-transition temperature $\left(v_{g}, T_{g}\right)$

$$
v_{f}=v_{g}\left[0.025+\alpha\left(T-T_{g}\right)\right]
$$

where $\alpha$ is the difference in the thermal expansion coefficients of the liquid and the glass state of the liquid. Combining Eqs. (4) and (5) gives the temperature dependence of viscosity. Cohen and Turnbull [155] provided a physical derivation of the Doolittle formula based on the idea that molecules' transport occurs when the sizes of some voids become greater than certain critical value and employed the Einstein relation between viscosity and diffusivity.

Poiseuille first observed that the addition of salts into water can increase or decrease viscosity [156]. Jones and Dole [157] developed correlation for the viscosity dependence of electrolytes as $\mu /$ $\mu_{o}=1+A c^{1 / 2}+B c$. Coefficient $A$ is always positive, but $B$ can be positive or negative [158], related to whether the ions are structure-making or structure-breaking.

Viscosity of mixtures of liquids is more complicated [159] and can vary nonmonotonically with composition, showing maximum and minimum in some mixtures while behaving monotonically in others. Different correlations are summarized in Ref. [159]. Volume and density are key parameters in these correlations, consistent with the free-volume idea.

In light of the success of the phonon picture in explaining the specific heat, one can question if the high-frequency phonons have any effects on viscosity. Andrade commented on the work of Brillouin [157], who hypothesized that the impacts of molecules create elastic waves in liquid. However, Brillouin's theory cannot explain experimental data. Molecular dynamics simulation of viscosity tends to underpredict viscosity measured experimentally by $\sim 40 \%$ [62]. Whether this underprediction is due to the collective resistance of phonons to viscous flow is a question worth answering.

\section{Summary and Potential Future Directions}

Based on the above discussion, it is fair to say that our current understanding of fundamental molecular pictures of the thermophysical properties of liquids is very unsatisfactory. It is also fair to say that there are good opportunities to make progress in this area by leveraging what has been done in the past.

On the basic pictures of thermal transport, it is my view that the traditional statistical approach based on the radial and pair- distribution functions and the reduction of the particle distribution functions to lower order will not be fruitful. The classical statistical approach worked reasonably well for the hydrodynamic transport, which does not need to access high-frequency modes. However, specific heat and thermal conductivity require us to consider high-frequency modes. Frenkel's phonon picture, as championed recently by Trachenko and co-workers, seems to be a more fruitful direction. The instantaneous normal-mode analysis and the two-phase model provide complementary pictures to the phonon model, as well as Eyring's significant structure theory. Further reconciling these pictures may be fruitful. Even the older lattice and free-volume models have some consistency with the phonon picture.

Although Trachenko's theory can explain specific heat of simple liquids, it cannot explain more complex liquids because it has neglected internal modes of molecules, which move toward lower frequency as more atoms are added to the molecule and become more accessible to thermal excitation. Extension of Trachenko's model to include these internal modes could be done without much difficulty, at least to molecules of moderate molecule length. However, very long molecules create complicated internal structures that will complicate the mode analysis [92].

The thermal conductivity is more difficult to model. No current models can capture all temperature dependence trends of liquids. All kinetic pictures have resorted to assuming energy transport happens across interatomic distance. This is fundamentally in conflict with the phonon picture, for which a more reasonable assumption is that energy can travel half wavelength [161]. Since for quite a few liquids the specific heat trend is different from the thermal conductivity trend in terms of their temperature dependence, using the current kinetic models, the only other variable is the speed of sound. It is unlikely that this will be able to explain the thermal conductivity trends. In fact, why all energy is carried by the speed of sound is a worthy question to ask. Like what we have mentioned with regard to the internal modes in the molecules in discussing specific heat, proper models should include heat conduction within the molecule as well as across molecules. Molecular dynamics simulation could provide valuable insights. More work needs to be done in terms of methods to analyze contributions of different modes. Some current research on heat conduction in amorphous materials can be useful for such purposes [118]. Insights might be gained from applying concepts such as diffuson, propagon, and locon to liquids [162]. In fact, I believe that liquid might be easier to understand than amorphous materials because structures of liquid should be close to equilibrium, which is not the case in amorphous materials. There are also efforts in formulating heat conduction from first principles to include disordered systems [163,164], leveraging recent advances in first-principles simulations $[7,165]$. Whether these formulations could be applied to liquids remains to be seen.

Viscosity modeling has better success than thermal conductivity, because the hydrodynamic and free-volume pictures are more suitable for fluid flow. However, it is valid to ask if the phonon pictures, the higher frequency modes, can affect viscosity. This question still needs to be clarified.

Many heat transfer systems desire higher thermal conductivity liquids. Although pressure and temperature can influence thermal conductivity, their values are determined by system operation conditions. Other external methods to engineer thermal conductivity of liquids are desirable. Addition of salt such as $\mathrm{NaF}$ had led to thermal conductivity higher than water. I have hypothesized the reason for the increment may be because both $\mathrm{Na}^{+}$and $\mathrm{F}^{-}$ions are structure makers. There might be other structure-making ions that can be developed to improve liquids thermal conductivity, such as hydroxyl ions $\mathrm{OH}^{-}$. Mixtures so far have shown thermal conductivity falling in between the two pure components. Thermal conductivity of papaya pulp was reported to be $0.65 \mathrm{~W} / \mathrm{m} \mathrm{K}$ at $20^{\circ} \mathrm{C}$, higher than pure water [166]. However, it is not clear this high value is due to experimental error. Many researchers had pursued the strategy of adding nanoparticles into liquids to 
increase their thermal conductivity, which I will not discuss here but refer readers to some of our publications discussing the heat conduction mechanisms [167].

\section{Acknowledgment}

I would like to thank Professor Ayyaswamy for his kind invitation and his critical reading of the paper. I would like to thank Professor Taku Ohara for clarifying some of my questions on his work and his comments on the paper, Mr. Simo Pajovic and Mr. Lenan Zhang for carefully proof-reading the paper, and Ms. Elizabeth Saas for language editing. I would like to thank MIT for its support at a difficult time.

\section{References}

[1] Vincenti, W. G., and Kruger, C. H., 1986, Introduction to Physical Gas Dynamics, Krieger, Malabar, FL, pp. 325-348

[2] Chen, G., 2005, Nanoscale Energy Transport and Conversion: A Parallel Treatment of Electrons, Molecules, Phonons, and Photons, Oxford University Press, Oxford, UK.

[3] Cahill, D. G., Ford, W. K., Goodson, K. E., Mahan, G. D., Majumdar, A., Maris, H. J., Merlin, R., and Phillpot, S. R., 2003, "Nanoscale Thermal Transport," J. Appl. Phys., 93(2), pp. 793-818.

[4] Cahill, D. G., Braun, P. V., Chen, G., Clarke, D. R., Fan, S. H., Goodson, K E., Keblinski, P., et alet al., 2014, "Nanoscale Thermal Transport. II. 20032012," Appl. Phys. Rev., 1(1), p. 011305.

[5] Chen, G., 2021, "Non-Fourier Phonon Heat Conduction at the Microscale and Nanoscale," Nat. Rev. Phys., 3(8), pp. 555-569.

[6] Qian, X., Zhou, J. W., and Chen, G., 2021, "Phonon-Engineered Extreme Thermal Conductivity Materials," Nat. Mater., 20(9), pp. 1188-1202.

[7] Broido, D. A., Malorny, M., Birner, G., Mingo, N., and Stewart, D. A., 2007, "Intrinsic Lattice Thermal Conductivity of Semiconductors From First Principles," Appl. Phys. Lett., 91(23), p. 231922.

[8] Peierls, R., 1929, "Zur Kinetischen Theorie der Warmeleitung in Kristallen," Ann. Phys., 395(8), pp. 1055-1101.

[9] Enskog, D., 1922, "Kinetische Theorie der Warmeleitung, Reibung und Selbstdiffusion in Gerwissen Verdichteten Gasen und Flussigkeiten." Kungliga Svenska Vatenskapsakademiens Handlingar, Ny Föld, 63(4), pp. 3-44.

[10] Einstein, A., 1905, "On the Movement of Small Particles Suspended in a Stationary Liquid Demanded by the Molecular Kinetic Theory of Heat," Ann. Phys., 322(8), pp. 549-560.

[11] Hansen, J.-P., and McDonald, I. R., 2013, Theory of Simple Liquids With Applications to Soft Matter, 4th ed., Elsevier, Amsterdam, The Netherlands.

[12] Frenkel, J., 1947, Kinetic Theory of Liquids, Oxford University Press, Oxford, UK.

[13] Maxwell, J. C., 1867, "On the Dynamic Theory of Gases," Philos. Trans., 157(5), pp. 49-88.

[14] Trachenko, K., and Brazhkin, V. V., 2016, "Collective Modes and Thermodynamics of the Liquid State," Rep. Prog. Phys., 79(1), p. 016502.

[15] Boon, J. P., and Yip, S., 1991, Molecular Hydrodynamics, Dover, New York.

[16] Lemmon, E. W., McLinden, M. O., and Friend, D. G., "Thermophysical Properties of Fluid Systems," NIST Chemistry WebBook, NIST Standard Reference Database Number 69, P. J. Linstrom and W. G. Mallard, eds., National Institute of Standard and Technology, Gaithersburg, MD, accessed Oct. 21, 2021, https://doi.org/10.18434/T4D303

[17] Vargaftik, N. B., Filippov, L. P., Tarzimanov, A. A., and Totskii, E. E., 1994, Handbook of Thermal Conductivity of Liquids and Gases, CRC Press, Boca Raton, FL.

[18] Yaws, C. L., 2009, Transport Properties of Chemicals and Hydrocarbons: Viscosity, Thermal Conductivity, and Diffusivity of C1 to C100 Organics and Ac to Zr Inorganics, Andrew William, Norwich, NY, pp. 299-402.

[19] Poling, B. E., Prausnitz, J. M., and O'Connell, J. P., 2001, The Properties of Gases and Liquids, 5th ed., McGraw-Hill, New York, pp. 10.42-10.56.

[20] Baroncini, C., Filippo, P. D., Latini, G., and Pacetti, M., 1981, "Organic Liquid Thermal Conductivity: A Prediction Method in the Reduced Temperature Range 0.3 to 0.8 ," Int. J. Thermophys., 2(1), pp. 21-38.

[21] Baroncini, C., Latini, G., and Pierpaoli, P., 1984, "Thermal Conductivity of Organic Liquid Binary Mixtures: Measurements and Prediction Method," Int. J. Thermophys., 5(4), pp. 387-401.

[22] Chen, H. S., 1980, "Glassy Metals," Rep. Prog. Phys., 43(4), pp. 353-432.

[23] Green, H. S., 1960, "Structure of Liquids," Encyclopedia of Physics: Structure of Liquids, S. Flugge, ed., Springer-Verlag, Berlin, pp. 1-133.

[24] Barker, J. A., and Henderson, D., 1976, "What Is "Liquid"? Understanding the States of Matter," Rev. Mod. Phys., 48(4), pp. 587-671.

[25] Lennard-Jones, J. E., and Devonshire, A. F., 1937, "Critical Phenomena in Gases-I," Proc. R. Soc. London A, 163(912), pp. 53-70.

[26] Lennard-Jones, J. E., and Devonshire, A. F., 1938, "Critical Phenomena in Gases II. Vapour Pressures and Boiling Points," Proc. R. Soc. London A, 165(920), pp. 1-11.

[27] Lennard-Jones, J. E., and Devonshire, A. F., 1939, "Critical and Co-Operative Phenomena III. A Theory of Melting and Structure of Liquids," Proc. R. Soc. London A, 169(938), pp. 317-338.
[28] Eyring, H., and Hirschfelder, J., 1937, "The Theory of the Liquid States," J. Phys. Chem., 41(2), pp. 249-257.

[29] Hirschfelder, J., Stevenson, D., and Eyring, H., 1937, “A Theory of Liquid Structure," J. Chem. Phys., 5(11), pp. 896-912.

[30] Frank, H. S., 1945, "Free Volume and Entropy in Condensed Systems," J. Chem. Phys., 13(11), pp. 478-492.

[31] Jhon, M. S., and Eyring, H., 1971, "The Significant Structure Theory of Liquids," Physical Chemistry-An Advanced Treatise: Liquid State, H. Eyring, D. Henderson, and W. Jost, eds., Academic Press, New York, pp. 335-375.

[32] Flory, P. J., 1953, Principles of Polymer Chemistry, Cornell University Press, Ithaca, NY.

[33] Hildebrand, J. H., 1947, "The Entropy of Solution of Molecules of Different Size," J. Chem. Phys., 15(5), pp. 225-228.

[34] Bogoliubov, N. N., 1946, "Kinetic Theory," J. Phys. USSR, 16(8), pp. 691-702.

[35] Born, M., and Green, H. S., 1946, "A General Kinetic Theory of Liquids, I. The Molecular Distribution Functions,” Proc. R. Soc. London A: Math. Phys. Sci., 188(1012), pp. 11-18.

[36] Kirkwood, J. G., 1946, "The Statistical Mechanical Theory of Transport Processes," J. Chem. Phys., 14(3), pp. 180-201.

[37] Yvon, J., and Rocard, Y., 1935, La Theorie Statistique des Fluides et L'equation d'etat, Hermann \& Cie, Paris, France.

[38] Ornstein, L. S., and Zernike, F., 1914, "Accidental Deviations of Density and Opalescence at the Critical Point of a Single Substance," KNAW, Proc., 17(2), pp. 793-806.

[39] Percus, J. K., and Yevick, G. J., 1958, “Analysis of Classical Statistical Mechanics by Means of Collective Coordinates," Phys. Rev., 110(1), pp. $1-12$.

[40] Langevin, P., 1908, "Sur la Theorie du Mouvement Brownien," C. R. Acad. Sci. (Paris), 146(3), pp. 530-533 [Lemons, D. S., and Cythiel, A., Am. J. Phys. 65(11), 1079 (1997)

[41] Smoluchowski, M., 1906, "Zur Kinetischen Theorie der Bronschen Molekularbewegung und der Suspensionen,” Ann. Phys., 326(14), pp. 756-780.

[42] Chandrasekhar, S., 1943, "Stochastic Problems in Physics and Astronomy," Rev. Mod. Phys., 15(1), pp. 1-89.

[43] Landau, L. D., and Lifshitz, E. M., 1959, Fluid Mechanics, Pergamon Press, London.

[44] Hauge, E. H., and Martin-Lof, A., 1973, "Fluctuating Hydrodynamics and Brownian Motion," J. Stat. Phys., 7(3), pp. 259-280.

[45] Radhakrishan, R., Ramakrishnan, N., Eckmann, D. M., and Ayyaswamy, P. S. 2020, "Nanoscale Fluid Dynamics," 21 st Century Nanoscience-A Handbook, 1 st ed., K. D. Sattler, ed., Taylor \& Francis, CRC Press, Boca Raton, FL.

[46] Radhakrishnan, R., Farokhirad, S., Eckmann, D. M., and Ayyaswamy, P. S. 2019, "Nanoparticle Transport Phenomena in Confined Flows," Adv. Heat Transfer, 51, pp. 55-129.

[47] Vitoshkin, H., Yu, H.-Y., Eckmann, D. M., Ayyaswamy, P. S., and Radhakrishnan, R., 2016, "Nanoparticle Stochastic Motion in the Inertial Regime and Hydrodynamic Interactions Close to a Cylindrical Wall," Phys. Rev. Fluids, 1(5), p. 054104.

[48] Yu, H.-Y., Eckmann, D. M., Ayyaswamy, P. S., and Radhakrishnan, R., 2015, "Composite Generalized Langevin Equation for Brownian Motion in Different Hydrodynamic and Adhesion Regimes," Phys. Rev. E, 91(5), p. 052303.

[49] Ramakrishnan, N., Wang, Y., Eckmann, D. M., Ayyaswamy, P. S., and Radhakrishnan, R., 2017, "Motion of a Nano-Spheroid in a Cylindrical Vessel Flow: Brownian and Hydrodynamic Interactions," J. Fluid Mech., 821, pp. $117-152$.

[50] Uma, B., Swaminathan, T. N., Radhakrishnan, R., Eckmann, D. M., and Ayyaswamy, P. S., 2011, "Nanoparticle Brownian Motion and Hydrodynamic Interactions in the Presence of Flow Fields," Phys. Fluids, 23(7), p. 073602.

[51] Radhakrishnan, R., Yu, H.-Y., Eckmann, D. M., and Ayyaswamy, P. S., 2017 "Computational Models for Nanoscale Fluid Dynamics and Transport Inspired by Non-Equilibrium Thermodynamics," ASME J. Heat Transfer-Trans. ASME, 139(3), p. 033001.

[52] Uma, B., Eckmann, D. M., Ayyaswamy, P. S., and Radhakrishnan, R., 2012 "A Hybrid Formalism Combining Fluctuating Hydrodynamics and Generalized Langevin Dynamics for the Simulation of Nanoparticle Thermal Motion in an Incompressible Fluid Medium," Mol. Phys., 110(11-12), pp. 1057-1067.

[53] Born, M., and Green, H. S., 1947, "A Kinetic Theory of Liquids," Nature 159(4034), pp. 251-254.

[54] Irving, J. H., and Kirkwood, J. G., 1950, "The Statistical Mechanical Theory of Transport Processes. IV. The Equation of Hydrodynamics," J. Chem. Phys., 18(6), pp. 817-829.

[55] Alder, B. J., and Wainwright, T. E., 1970, "Decay of the Velocity Autocorrelation Function," Phys. Rev. A, 1(1), pp. 18-21.

[56] Mori, H., 1965, "Transport, Collective Motion, and Brownian Motion," Prog. Theor. Phys., 33(3), pp. 423-455.

[57] Widom, A., 1971 "Velocity Fluctuation of a Hard-Core Brownian Particle," Phys. Rev. A, 3(4), pp. 1394-1396.

[58] Car, R., and Parrinello, M., 1985, "Unified Approach for Molecular Dynamics and Density-Functional Theory," Phys. Rev. Lett., 55(22), pp. 2471-2474.

[59] Green, M. S., 1952, "Markoff Random Processes and the Statistical Mechanics of Time-Dependent Phenomena," J. Chem. Phys., 20(8), pp. 1281-1295.

[60] Kubo, R., 1957, "Statistical-Mechanical Theory of Irreversible Processes. I General Theory and Simple Applications to Magnetic and Conduction Problems," J. Phys. Soc. Jpn., 12(6), pp. 570-586.

[61] Hardy, R. J., 1963, "Energy-Flux Operator for a Lattice," Phys. Rev., 132(1), pp. 168-177. 
[62] Ungerer, P., Nieto-Draghi, C., Rousseau, B., Ahunbay, G., and Lachet, V., 2007, "Molecular Simulation of the Thermophyical Properties of Fluids: From Understanding Towards Quantitative Predictions," J. Mol. Liq., 134(1-3), pp. 71-89.

[63] Frenkel, J., 1926, "Thermal Movement in Solid and Liquid Bodies," Z. Physik, 35, 652-669.

[64] Bolmatov, D., Brazhkin, V. V., and Trachenko, K., 2012, "The Phonon Theory of Liquid Thermodynamics," Sci. Rep., 2(1), p. 421.

[65] Crimsditch, M., Bhadra, R., and Torell, L. M., 1989, "Shear Waves Through the Glass-Liquid Transformation," Phys. Rev. Lett., 62, 2616-2619.

[66] Giordano, V. M., and Monaco, G., 2010, "Fingerprints of Order and Disorder on High-Frequency Dynamics of Liquids," PNAS, 107(51), pp. 21985-21989.

[67] Prigogine, I., Trappeniers, N., and Mathot, V., 1953, "Statistical Thermodynamics of r-MERS and r-MER Solutions," Discuss. Faraday Soc., 15, pp. 93-107.

[68] Seeley, G., and Keyes, T., 1989, "Normal-Mode Analysis of Liquid-State Dynamics,” J. Chem. Phys., 91(9), pp. 5581-5586.

[69] Buchner, M., Ladanyi, B. M., and Stratt, R. M., 1992, "The Short-Time Dynamics of Molecular Liquids. Instantaneous-Normal-Mode Theory," J. Chem. Phys., 97(11), pp. 8522-8535.

[70] Cho, M. H., Fleming, G. R., Saito, S., Ohmine, I., and Stratt, R. M., 1994 "Instantaneous Normal Mode Analysis of Liquid Water," J. Chem. Phys. 100(9), pp. 6672-6683.

[71] Gezelter, J. D., Rabani, E., and Berne, B. J., 1997, "Can Imaginary Instantaneous Normal Mode Frequencies Predict Barriers to Self-Diffusion," J. Chem. Phys., 107(12), pp. 4618-4627.

[72] Keyes, T., Li, W.-X., and Zurcher, U., 1998, "Comment on a Critique of the Instantaneous Normal Mode (INM) Approach to Diffusion [J. Chem. Phys. 107, 4618 (1997)]," J. Chem. Phys., 109(11), pp. 4693-4694.

[73] Lin, S.-T., Blanco, M., and Goddard, W. A., III, 2003, "The Two-Phase Model for Calculating Thermodynamic Properties of Liquids From Molecular Dynamics: Validation for the Phase Diagram of Lennard-Jones Fluids," J. Chem. Phys., 119(22), pp. 11792-11805.

[74] Lin, S.-T., Maiti, P. K., and Goddard, W. A., III, 2010, "Two-Phase Thermodynamic Model for Efficient and Accurate Absolute Entropy of Water From Molecular Dynamics Simulations," J. Phys. Chem. B, 114(24), pp. 8191-8198.

[75] Iwashita, T., Nicholson, D. M., and Egami, T., 2013, "Elementary Excitation and Crossover Phenomenon in Liquids," Phys. Rev. Lett., 110(20), p. 205504.

[76] Stillinger, F. H., 1980, “Water Revisited," Science, 209(4455), pp. 451-457.

[77] Ludwig, R., 2001, "Water: From Clusters to the Bulk," Angew. Chem. Int Ed., 40(10), pp. 1808-1827.

[78] Benson, S. W., and Siebert, E. D., 1992, "A Simple Two-Structure Model for Liquid Water,” J. Am. Chem. Soc., 114, pp. 4269-4276.

[79] Chaplin, M. F., 1999, "A Proposal for the Structuring of Water," Biophys. Chem., 83, pp. 211-221.

[80] Weinhold, F., 1998, "Quantum Cluster Equilibrium Theory of Liquids: General Theory and Computer Implementation," J. Chem. Phys., 109, pp. 367-372.

[81] Frank, H. S., and Wen, W.-Y., III, 1957, "Ion-Solvent Interaction, Structural Aspects of Ion-Solvent Interaction in Aqueous Solutions: A Suggested Picture of Water Structure," Discuss. Faraday Soc., 24, pp. 133-140.

[82] DeBye, P., and Huckel, E., 1923, "The Theory of Electrolyte. I. Freezing Point Depression and Related Phenomena (Zur Theorie der Elektrolyte. I. Gefrierpunktserniedrigung und Uermandte Erscheinungen)," Phys. Z., 24, pp. 185-206 (translated by M. J. Braus).

[83] Chen, G., "Donnan Equilibrium Revisited: Coupling Between Ion Concentration, Osmotic Pressure, and Donnan Potential,“" (to appear in J. Micromechanics \& Molecular Phys.).

[84] Eigen, M., and Wicke, E., 1954, "The Thermodynamics of Electrolytes at Higher Concentrations," J. Phys. Chem., 58(9), pp. 702-714.

[85] Brillouin, L., 1937, "On Thermal Agitation in Liquids," J. Phys., 33(9), pp. $54-55$.

[86] Wallace, D. C., 1998, "Liquid Dynamics Theory of High-Temperature Specific Heat," Phys. Rev. E, 57(2), pp. 1717-1722.

[87] Trachenko, K., 2008, "Heat Capacity of Liquids: An Approach From the Solid-Phase," Phys. Rev., 78, p. 104201

[88] Brazhkin, V. V., and Trachenko, K., 2014, "Collective Excitations and Thermodynamics of Disordered State: New Insights into an Old Problem," J. Phys. Chem. B, 118, 11417-11427.

[89] Lilley, D., Jain, A., and Prasher, R., 2021 "A Simple Model for the Entropy of Melting of Monatonic Liquids," Appl. Phys. Lett., 118, p. 083902.

[90] Pascal, T. A., Lin, S.-T., and Goddard, W. A., III, 2011, "Thermodynamics of Liquids: Standard Molar Entropies and Heat Capacity of Common Solvents From 2PT Molecular Dynamics," Phys. Chem. Chem. Phys., 13(1), pp. 169-181.

[91] Flory, P. J., Orwoll, R. A., and Vrij, A., 1964, "Statistical Thermodynamics of Chain Molecule Liquids. I. An Equation of State for Normal Paraffin Hydrocarbons," J. Am. Chem. Soc., 86(17), pp. 3507-3514.

[92] McLaughlin, E., 1964, "The Thermal Conductivity of Liquids and Dense Gases," Chem. Rev., 64(4), pp. 389-428.

[93] Touloukian, Y. S., Liley, P. E., and Saxena, S. C., 1970, Thermal Conductivity - Nonmetallic Liquids and Gases (Thermophysical Properties of Matter, The TPRC Data Series, Vol. 3), Y. S. Touloukian and C. Y. Ho, eds., Purdue University, West Lafayette, IN.

[94] Chapman, S., and Cowling, T. G., 1953, The Mathematical Theory of NonUniform Gases, Cambridge University Press, London.
[95] Bridgman, P. W., 1923, "The Thermal Conductivity of Liquids Under Pressure,” Proc. Am. Acad. Arts Sci., 59(7), pp. 141-169.

[96] Sakiadis, B. C., and Coates, J., 1955, "Studies of Thermal Conductivity of Liquids," AIChE J., 1(3), pp. 275-288.

[97] Sakiadis, B. C., and Coates, J., 1956, "Prediction of Specific Heat or Organic Liquids," AIChE J., 2(1), pp. 88-93.

[98] Horrocks, J. K., and McLaughlin, E., 1960, "Thermal Conductivity of Simple Molecules in the Condensed State," Trans. Faraday Soc., 56, pp. 206-212.

[99] Xi, Q., Zhong, J. X., He, J. X., Xu, X. F., Nakayama, T., Wang, Y. Y., Liu, J. Zhou, J., and Li, B. W., 2020, "A Ubiquitous Thermal Conductivity Formula for Liquids, Polymer Glass, and Amorphous Solids,” Chin. Phys. Lett., 37(10), p. 104401.

[100] Longuet-Higgins, H. C., and Pople, J. A., 1956, "Transport Properties of a Dense Fluid of Hard Spheres," J. Chem. Phys., 25(5), pp. 884-889.

[101] Longuet-Higgins, H. C., and Valleau, J. P., 1958, "Transport Coefficients of Dense Fluids of Molecules Interacting According to a Square Well Potential,' Mol. Phys., 1(3), pp. 284-294.

[102] Davis, H. T., Rice, S. A., and Sengers, J. V., 1961, "On the Kinetic Theory of Dense Fluids. IX. The Fluid of Rigid Spheres With a Square-Well Attraction," J. Chem. Phys., 35(6), pp. 2210-2233.

[103] Rice, S. A., and Kirkwood, J. G., 1959, "On an Approximate Theory of Transport in Dense Media," J. Chem. Phys., 31(4), pp. 901-908.

[104] Rice, S. A., and Allnatt, A. R., 1961, "On the Kinetic Theory of Dense Fluids. VI. Singlet Distribution Function for Rigid Spheres With an Attractive Potential," J. Chem. Phys., 34(6), pp. 2144-2155.

[105] Naghizadeh, J., and Rice, S. A., 1962, "Kinetic Theory of Dense Fluids. X. Measurement and Interpretation of Self-Diffusion in Liquid $\mathrm{Ar}, \mathrm{Kr}, \mathrm{Xe}$, and $\mathrm{CH}_{4}$," J. Chem. Phys., 36(10), pp. 2710-2720.

[106] Ikenberry, L. D., and Rice, S. A., 1963, "On the Kinetic Theory of Dense Fluids. XIV. Experimental and Theoretical Studies of Thermal Conductivity in Liquid Ar, Kr, Xe, and $\mathrm{CH}_{4}$," J. Chem. Phys., 39(6), pp. 1561-1671.

[107] Eisenschitz, R., 1949, "The Steady Non-Uniform State for a Liquid," Proc. Phys. Soc. A, 62(1), pp. 41-49.

[108] Lin, S. H., Eyring, H., and Davis, W. J., 1964, "Thermal Conductivity of Liquids," J. Phys. Chem., 68(10), pp. 3017-3020.

[109] Callaway, J., 1959, "Model for Lattice Thermal Conductivity at Low Temperatures," Phys. Rev., 113(4), pp. 1046-1051.

[110] Klemens, P. G., 1955, "The Scattering of Low-Frequency Lattice Waves by Static Imperfections," Proc. Phys. Soc., Sec. A, 68(12), pp. 1113-1128.

[111] Zhao, A. Z., Wingert, M. C., Chen, R. K., and Garay, J. E., 2021, "Phonon Gas Model for Thermal Conductivity of Dense, Strongly Interacting Liquids," J. Appl. Phys., 129(23), p. 235101.

[112] Trachenko, K., Baggioli, M., Behnia, K., and Brazhkin, V. V., 2021, "Universal Lower Bounds on Energy and Momentum Diffusion in Liquids," Phys. Rev. B, 103(1), p. 014311.

[113] Owen, E. J., and Thodos, G., 1957, "Thermal-Conductivity-Reduced-State Correlation for the Inert Gas," AIChE J., 3(4), pp. 454-461.

[114] Trachenko, K., Monserrat, B., Pickard, C. J., and Brazhkin, V. V., 2020, "Speed of Sound From Fundamental Physical Constants," Sci. Adv., 6(41), p. eabc9622.

[115] Trachenko, K., and Brazhkin, V. V., 2020, "Minimal Quantum Viscosity From Fundamental Physical Constants," Sci. Adv., 6(17), p. eaba3747.

[116] Zhang, M., Lussetti, E., de Souza, L. E. S., and Müller-Plathe, F., 2005, "Thermal Conductivity of Molecular Liquids by Reverse Nonequilibrium Molecular Dynamics," J. Phys. Chem., 109(31), pp. 15060-15067.

[117] McGaughey, A. J. H., and Larkin, J. M., 2014, "Predicting Phonon Properties From Equilibrium Molecular Dynamics Simulations," Annu. Rev. Hea Transfer, 17, pp. 49-87.

[118] Lv, W., and Henry, A., 2016, "Direct Calculation of Modal Contributions to Thermal Conductivity Via Green-Kubo Modal Analysis," New J. Phys., 18(1), p. 013028.

[119] Wei, X. F., Wang, Z., Tian, Z. T., and Luo, T. F., 2021, "Thermal Transport in Polymers: A Review," ASME J. Heat Transfer-Trans. ASME, 143(7), p. 072101 .

[120] Matsubara, H., Kikugawa, G., Ishikiriyama, M., Yamashita, S., and Ohara, T., 2017, "Equivalence of the EMD- and NEMD-Based Decomposition of Thermal Conductivity Into Microscopic Building Blocks," J. Chem. Phys., 147(11), p. 114104

[121] Torii, D., Nakano, T., and Ohara, T., 2008, "Contribution of Inter- and Intramolecular Energy Transfers to Heat Conduction in Liquids," J. Chem. Phys., 128(4), p. 044504.

[122] Surblys, D., Matsubara, H., Kikugawa, G., and Ohara, T., 2019, "Application of Atomic Stress to Compute Heat Flux Via Molecular Dynamics for Systems With Many-Body Interactions," Phys. Rev. E, 99(5), p. 05130(R).

[123] Ohara, T., 1999, "Contribution of Intermolecular Energy Transfer to Heat Conduction in a Simple Liquid," J. Chem. Phys., 111(21), pp. 9667-9672.

[124] Fleming, F. P., Silva, L. A., Lima, G. S. V., Herzog, I., Orlande, H. R. B., Daridon, J.-L., Pauly, J., and Azevedo, L. F. A., 2018, "Thermal Conductivity of Heavy, Even-Carbon Number n-Alkanes (C22 to C32)," Fluid Phase Equilib., 477, pp. 78-86.

[125] Huang, D. H., Simon, S. L., and McKenna, G. B., 2005, "Chain Length Dependence of the Thermodynamic Properties of Linear and Cyclic Alkanes and Polymers," J. Chem. Phys., 122(8), p. 084907.

[126] Henry, A., and Chen, G., 2008, "High Thermal Conductivity of Single Polyethylene Chains Using Molecular Dynamics Simulations," Phys. Rev. Lett., 101(23), p. 235502. 
[127] Ohara, T., Yuan, T. C., Torii, D., Kikugawa, G., and Kosugi, N., 2011, "Heat Conduction in Chain Polymer Liquids: Molecular Dynamics Studies on the Contributions of Inter- and Intramolecular Energy Transfer," J. Chem. Phys., 135(3), p. 034507.

[128] Matsubara, H., Kikugawa, G., Bessho, T., Yamashita, S., and Ohara, T., 2015, "Effects of Molecular Structure on Microscopic Heat Transport in Chain Polymer Liquids," J. Chem. Phys., 142(16), p. 164509.

[129] Matsubara, H., Kikugawa, G., Bessho, T., Yamashita, S., and Ohara, T., 2017, "Understanding the Chain Length Dependence of Thermal Conductivity of Liquid Alcohols at $298 \mathrm{~K}$ on the Basis of Molecular-Scale Energy Transfer," Fluid Phase Equilib., 441, pp. 24-32.

[130] Matsubara, H., Kikugawa, G., Ishikiriyama, M., Yamashita, S., and Ohara, T. 2018, "Microscopic Picture of Heat Conduction in Liquid Ethylene Glycol by Molecular Dynamics Simulation: Difference From the Monohydric Case," Int. J. Heat Mass Transfer, 121, pp. 1033-1038.

[131] Yang, F., Lu, H. X., Liu, W. Q., and Zhou, H., 2020 "Understanding the Contributions of Microscopic Heat Transfer to Thermal Conductivities of Liquid Aldehydes and Ketones by Molecular Dynamics Simulations," J. Chem. Inf Model., 60(6), pp. 3022-3029.

[132] Ohara, T., 1999, "Intermolecular Energy Transfer in Liquid Water and It Contribution to Heat Conduction: A Molecular Dynamics Study," J. Chem. Phys., 111(14), pp. 6492-6500.

[133] Sirk, T. W., Moore, S., and Brown, E. F., 2013, "Characteristics of Thermal Conductivity in Classical Water Models," J. Chem. Phys., 138(6), p. 064505.

[134] Palmer, G., 1948, “Thermal Conductivity of Liquids," Ind. Eng. Chem., 40(1), pp. 89-92.

[135] Wang, P. M., and Anderko, A., 2008, "Modeling Thermal Conductivity of Concentrated and Mixed-Solvent Electrolyte Systems," Ind. Eng. Chem. Res. 47(15), pp. 5698-5709.

[136] Hamer, W. J., and Wu, Y.-C., 1972, "Osmotic Coefficients and Mean Activity Coefficients of Uni-Univalent Electrolytes in Water at $25^{\circ} \mathrm{C}$," J. Phys. Chem. Ref. Data, 1(4), pp. 1047-1099.

[137] Fortier, J. L., Philip, P. R., and Desnoyers, J. E., 1974, “Thermodynamic Properties of Alkali Halides. III. Volumes and Heat Capacities of Transfer From $\mathrm{H}_{2} \mathrm{O}$ to $\mathrm{D}_{2} \mathrm{O}$ at $25^{\circ} \mathrm{C}$," J. Solution Chem., 3(7), pp. 523-537.

[138] Robertson, R. E., Sugamor, S. E., Tse, R., and Wu, C.-Y., 1966, "The Solvent Isotope Effect and the Partial Molar Volume of Ions," Can. J. Chem., 44(4), pp. 487-494

[139] Glueckauf, E., 1968, "Molar Volumes of Ions, Part 2," Trans. Faraday Soc., 64, pp. 2423-2432.

[140] Huang, B. T., Muy, S., Feng, S. T., Katayama, Y., Lu, Y.-C., Chen, G., and Shao-Horn, Y., 2018, "Non-Covalent Interactions in Electrochemical Reactions and Implications in Clean Energy Applications," Phys. Chem. Chem. Phys., 20(23), pp. 15680-15686.

[141] Kontogeorgis, G. M., Maribo-Mogensen, B., and Thomsen, K., 2018, "The Debye-Huckel Theory and Its Importance in Modeling Electrolyte Solutions," Fluid Phase Equilib., 462, pp. 130-152.

[142] Liu, H. J., Maginn, E., Visser, A. E., Bridges, N. J., and Fox, E. B., 2012, "Thermal and Transport Properties of Six Ionic Liquids: An Experimental and Molecular Dynamics Study," IEC Res., 51(21), pp. 7242-7254.

[143] Grosse, A. V., 1966, "Electrical and Thermal Conductivity of Metals Over Their Entire Liquid Range,” Rev. Hautes Temp. Refract., 3(2), pp. 115-146.

[144] Mott, N. F., 1934, "The Resistance of Liquid Metals," Proc. R. Soc. A, 146(9), pp. 465-472.

[145] Ziman, J. M., 1961, "A Theory of the Electrical Properties of Liquid Metals. I The Monovalent Metals," Philos. Mag., 6(68), pp. 1013-1034.

[146] Sobolev, A. N., and Mirzoev, A. A., 2008, "The Kubo-Greenwood Calculation of Conductivity of the Simple and Non-Simple Liquid Metals in a Wide Temperature Range," J. Phys.: Conf. Ser., 98(6), p. 062015.
[147] Yu, L. Y., Hou, X. J., Wu, K. J., and He, C. H., 2021, "Measurements of the Thermal Conductivity of 1-Menthol-Decanoic Acid Deep Eutectic Solvents in the Temperature Range From 283.15 to $363.15 \mathrm{~K}$ at Pressures Up to 15.1 MPa," J. Chem. Eng. Data, 66(5), pp. 2061-2070.

[148] Bearman, R. J., 1958, "Statistical Mechanical Theory of Thermal Conductivity of Binary Liquid Solutions," J. Chem. Phys., 29(6), pp. 1278-1286.

[149] Bearman, R. J., Kirkwood, J. G., and Fixman, M., 1958, "StatisticalMechanical Theory of Transport Processes. X. The Heat of Transport in Binary Liquid Solutions," Adv. Chem. Phys., 1, pp. 1-13.

[150] Fang, Y. P., Kikugawa, G., Matsubara, H., Bessho, T., Yamashita, S., and Ohara, T., 2016, "Molecular Thermal Energy Transfer in Binary Mixture of Simple Liquids," Fluid Phase Equilib., 429, pp. 293-300.

[151] Andrade, E. N. D. C., 1934, "A Theory of the Viscosity of LiquidsPart I," London, Edingburg, Dublin Philos. Mag. J. Sci., 17(112), pp. 497-511.

[152] Eyring, H., 1936, "Viscosity, Plasticity, and Diffusion as Examples of Absolute Reaction Rates," J. Chem. Phys., 4(4), pp. 283-291.

[153] Doolittle, A. K., 1951, "Studies in Newtonian Flow. 2. The Dependence of the Viscosity of Liquids on Free-Space," J. Appl. Phys., 22(12), pp. $1471-1475$.

[154] Williams, M. L., Landel, R. F., and Ferry, J. D., 1955, "The Temperature Dependence of Relaxation Mechanisms in Amorphous Polymers and Other Glass-Forming Liquids," J. Am. Chem. Soc., 77(14), pp. 3701-3707.

[155] Cohen, M. H., and Turnbull, D., 1959, "Molecular Transport in Liquids and Glasses," J. Chem. Phys., 31(5), pp. 1164-1169.

[156] Poiseuille, J. L. M., 1847, "Sur le Mouvement des Liquides de Nature Differente dans les Tubes de Tres Petits Diametres," Ann. Chim. Phys., Ser. 3, 21, pp. 76-110.

[157] Jones, G., and Dole, M., 1929, "The Viscosity of Aqueous Solutions of Strong Electrolytes With Special Reference to Barium Chloride,” J. Am. Chem. Soc., 51(10), pp. 2950-2964.

[158] Jenkins, H. D. B., and Marcus, Y., 1995, "Viscosity B-Coefficients of Ions in Solution," Chem. Rev., 95(8), pp. 2695-2724.

[159] Irving, J. B., 1977, "Viscosities of Binary Liquid Mixtures: The Effectiveness of Mixture Equations," National Engineering Laboratory, East Kilbride, Glasgow, UK, Report No. 631.

[160] Brillouin, L., 1922, "La Viscosite Des Liquides Et Son Interpretation Theorique," J. Phys. Radium, 3(9), pp. 326-340.

[161] Cahill, D., Watson, S., and Pohl, R., 1992, "Lower Limit to the Thermal Conductivity of Disordered Crystals," Phys. Rev. B, 46(10), pp. 6131-6140.

[162] Allen, P. B., Feldman, J. L., Fabian, J., and Wooten, F., 1999, "Diffusons, Locons and Propagons: Character of Atomic Vibrations in Amorphous Si," Philos. Mag. B, 79(11-12), pp. 1715-1731.

[163] Simoncelli, M., Marzari, N., and Mauri, F., 2019, "Unified Theory of Thermal Transport in Crystals and Glasses," Nat. Phys., 15(8), pp. 809-813.

[164] Isaeva, L., Barbalinardo, G., Donadio, D., and Baroni, S., 2019, "Modeling Heat Transport in Crystals and Glasses From a Unified Lattice-Dynamical Approach," Nat. Commun., 10(1), p. 3853.

[165] Lindsay, L., Hua, C., Ruan, X. L., and Lee, S., 2018, "Survey of Ab Initio Phonon Thermal Transport," Mater. Today Phys., 7, pp. 106-120.

[166] Espinoza-Guevara, R., Caro-Corrales, J., Ordorica-Falomir, C., ZazuetaMorales, J., Vega-Garcia, M., and Cronin, K., 2010, "Thermophysical Properties of Pulp and Rind of Papaya Cv. Maradol," Int. J. Food Prop., 13(1), pp. 65-74

[167] Wang, J. J., Zheng, R. T., Gao, J. W., and Chen, G., 2012, "Heat Conduction Mechanisms in Nanofluids and Suspensions," Nano Today, 7(2), pp. 124-136. 